\title{
Phylogenetic profiling and cellular analyses of ARL16 reveal roles in traffic of IFT140 and INPP5E
}

Skylar I. Dewees ${ }^{1,2}$, Romana Vargováa ${ }^{3}$, Katherine R. Hardin ${ }^{2,4}$, Rachel E. Turn ${ }^{1,2,5}$, Saroja Devi ${ }^{1}$, Joshua Linnert $^{6}$, Uwe Wolfrum ${ }^{6}$, Tamara Caspary ${ }^{7}$, Marek Eliáš ${ }^{3}$, and Richard A. Kahn", \#

${ }^{1}$ Department of Biochemistry, Emory University School of Medicine, Atlanta, GA 30322

2 Biochemistry, Cell \& Developmental Biology Graduate Program, Laney Graduate School, Emory University

${ }^{3}$ Department of Biology and Ecology, Faculty of Science, University of Ostrava, Chittussiho 10, Ostrava, Czech Republic

${ }^{4}$ Department of Cell Biology, Emory University School of Medicine, Atlanta, GA 30322

${ }^{5}$ Department of Microbiology and Immunology, Stanford University, Palo Alto, CA, USA

${ }^{6}$ Institute of Molecular Physiology, Johannes Gutenberg University, Mainz 55128, Germany

${ }^{7}$ Department of Human Genetics, Emory University School of Medicine, Atlanta, GA 30322

\# Corresponding author: Richard A. Kahn, rkahn@emory.edu; Department of Biochemistry, Emory University School of Medicine, 1510 Clifton Rd, Atlanta, GA, USA 30322; Orcid \#0000-00020259-0601; rkahn@emory.edu

\section{Summary:}

Phylogenetic analyses of ARF family GTPases predict that ARL16 is linked to cilia. This was confirmed using MEFs deleted for ARL16, resulting in defects in Golgi to cilium traffic, with accumulation of IFT140 and INPP5E at Golgi.

Running head: ARL16 regulation of ciliary proteins

Abbreviations include: Ac-Tu, acetylated tubulin; GAP, GTPase activating protein; GEF, guanine nucleotide exchange factor; $\mathrm{KO}$, knock out cell line; MEF, mouse embryonic fibroblast.

\section{Key Words}

ARL16, primary cilia, ciliogenesis, Golgi, IFT140, INPP5E, PDE6D

\section{ABSTRACT}

The ARF family of regulatory GTPases is ancient, with 16 members predicted to have been present in the last eukaryotic common ancestor. Our phylogenetic profiling of paralogs in diverse species identified four family members whose presence correlates with that of a cilium/flagellum: ARL3, ARL6, ARL13, and ARL16. No prior evidence links ARL16 to cilia or other cell functions, despite its presence throughout eukaryotes. Deletion of ARL16 in MEFs results in decreased ciliogenesis yet increased ciliary length. We also found Arl16 KO in MEFs to alter ciliary protein content, including loss of ARL13B, ARL3, INPP5E, and the IFT-A core component IFT140. Instead, both INPP5E and IFT140 accumulate at the Golgi in Arl16 KO lines, while other IFT proteins do not, suggesting a specific defect in traffic from Golgi to cilia. We propose that ARL16 regulates a Golgi-cilia traffic pathway and is required specifically in the export of IFT140 and INPP5E from the Golgi. 


\section{INTRODUCTION}

ARF family regulatory GTPases are best known for their roles in regulating bi-directional membrane traffic throughout the secretory pathway, including at Golgi and endosomes (Sztul et al., 2019). However, they also regulate a diverse array of other pathways and functions, including at cilia (Fisher et al., 2020), mitochondria, lipid droplets, centrioles, midbodies, and rods and rings (Sztul et al., 2019). To achieve such widespread cellular regulation, mammals express as many as 30 ARF family GTPases, including 6 ARFs, 22 ARF-like (ARL), 2 SARs, and TRIM23. Like all regulatory GTPases, they switch between "inactive" (GDP-bound) and "active" (GTP-bound) conformations in response to the actions of guanine nucleotide exchange factors (GEFs) and GTPase activating proteins (GAPs). With regulatory roles in such diverse and essential cellular processes, ARF family members are implicated in a large number of pathologies including cancers, ciliopathies, hearing and vision impairments, idiopathic pulmonary fibrosis, and other maladies. Despite their importance to cell biology and human health, we lack substantial mechanistic details of their actions (Sztul et al., 2019). Indeed, some of the core family members remain virtually unstudied.

We recently reported the results of a taxonomically broad phylogenetic analysis of the ARF family in eukaryotes (Vargova et al., 2021). This study defines the set of family members ancestral for eukaryotes and describes 16 paralogs putatively present in the last eukaryotic common ancestor. We used our large phylogenetic dataset to look for the retention or loss of family members that are correlated with retention or loss of the cilium. Strikingly, as elaborated below, the phyletic pattern of ARL16 genes provided a hypothesis that its cellular function is relevant to cilia. A link between ARL16 and cilia also emerged in a CRISPR screen for regulators of the Hedgehog signalling pathway (Breslow et al., 2018). ARL16 was previously reported to be cytosolic and inhibit the function of the RIG-I protein in defense against RNA viruses (Yang et al., 2011). Also, two high-throughput datasets identified potential interacting partners of ARL16, including PDE6D (aka PDE6 $\delta$ or PrBP/delta) and GM130, providing additional clues to its location and functions (Luck et al., 2020; Rolland et al., 2014). Thus, despite some fragmentary data on ARL16 from several systems there has been no systematic approach aimed at identifying fundamental actions of ARL16 in cells, or more specifically in cilia.

The primary cilium is a sensory structure, responding to extracellular signals with cellular responses through complex intracellular signalling networks. Primary cilia are made up of a microtubule-based backbone, the axoneme, which extends from the modified mother centriole, termed the basal body (Satir and Christensen, 2007). The axoneme is covered in the ciliary sheath, which is continuous with the plasma membrane but functionally separated from it by the transition zone, which tightly regulates entry to the ciliary compartment (Stephen et al., 2017). Three paralogs in the ARL family, ARL3, ARL6, and ARL13, play roles in ciliary functions, acting both inside and outside of cilia (Chiang et al., 2004; Fan et al., 2004; Fisher et al., 2020; Gigante and Caspary, 2020; Zhou et al., 2006). ARL3 regulates the delivery of N-myristoylated and prenylated proteins to the cilium by binding cargo transporters UNC119 and PDE6D, respectively (Fansa et al., 2016; Stephen et al., 2017). ARL6/BBS3 regulates the function of the BBSome (a protein complex involved in export of ciliary membrane proteins (Mourão et al., 2014). ARL13, including the extensively studied mammalian paralog ARL13B, is involved in ciliary protein import and export, partly mediated by its activity as a GEF for ARL3 (Gotthardt et al., 2015; Ivanova et al., 2017). It is also well known for its crucial role in vertebrate development via one of the best-known ciliary signaling pathways, Hedgehog (Hh) (Caspary et al., 2007). In this pathway, Hh ligand binds to its receptor, Patched $(\mathrm{PTCH})$ that is enriched on the ciliary membrane. This induces the removal of PTCH from the cilium, allowing the G protein coupled receptor (GPCR) Smoothened (SMO) to enter (Goetz et al., 2009). This leads to cleavage and activation of Gli transcription factors which then translocate to the nucleus, resulting in the transcription of target genes. This pathway is heavily influenced by other ciliary proteins including ARL13B, ARL3, and INPP5E, often through alterations in SMO recruitment or changes in Gli activation (Caspary et al., 2007; Jacoby et al., 2009; Lai et al., 2011). Changes in lipid metabolism are also likely involved as ARL13B directly binds both INPP5E (Qiu et al., 2021), a phosphoinositide 5'-phosphatase, and ARL3, to activate it and thereby promote release of INPP5E from its transporter PDE6D.

Coordinated protein traffic to and within the cilium is essential for both its formation (ciliogenesis) and function (ciliary signaling). Small proteins $(<\sim 100 \mathrm{kDa})$ can diffuse across the barrier at the base of the cilium, the transition zone (Dishinger et al., 2010; Nachury and Mick, 2019). The passage of other proteins through the 
transition zone is tightly controlled by the IFT protein complexes, IFT-A and IFT-B, and the BBSome (Lechtreck, 2015; Wingfield et al., 2018). Cilium assembly and length are regulated in large part by intraflagellar transport (IFT) machinery, with IFT-A and IFT-B complexes involved in retrograde and anterograde traffic, respectively (Nachury, 2018; Wang et al., 2021). These multi-subunit complexes are required for the regulated entry and export of proteins from cilia, acting in concert with another protein complex, the BBSome, at the base of cilia in the transition zone. IFT-A is also required for efficient traffic of ciliary membrane cargos to the cilium, acting as a vesicle coat complex between the Golgi and cilia (Quidwai et al., 2021). While the core and peripheral protein components of IFT-A and IFT-B have been established (e.g., IFT-A is comprised of Ciliary protein content is further regulated by targeting of newly synthesized proteins from the ER to the Golgi to the cilium and recycling of membrane proteins from the plasma membrane to the ciliary membrane either through lateral diffusion or recycling through endosomes, though these pathways are less well understood than traffic within the cilium itself (Carter and Blacque, 2019; Nachury, 2018).

To begin testing the hypothesis that ARL16 is important to ciliary biology and to search for additional roles in cell biology we knocked out the gene in immortalized mouse embryonic fibroblasts (MEFs). These lines display defects in ciliogenesis, ciliary protein traffic, and ciliary signalling, confirming the phylogenetic prediction and experimentally linking a fourth $A R L$ to cilia. Furthermore, the ciliary traffic defects observed in Arl16 KO cells appear to be linked to, and possibly the direct result of, changes in traffic of key ciliary proteins through the Golgi. Thus, these data provide an initial characterization of the cellular functions of ARL16 linked to cilia, as well as a role in protein export from the Golgi.

\section{RESULTS}

\section{ARL16 is a divergent member of the ARF family}

ARL16 is among the least characterized GTPases in the ARF family despite its predicted presence in the last eukaryotic common ancestor (Vargova et al., 2021). Sequence alignments reveal ARL16 to be among the most divergent members of the family in mammals, sharing only $\sim 27 \%$ identity to ARF1 (compared to $>60 \%$ identity among the ARFs and $>40 \%$ identity among most ARLs) (Fig. S1). Mammalian tissues widely express ARL16 mRNAs, based on searches of RNA and proteome databases, including NCBI Gene and GTexPortal. ARL16's length is unusually variable amongst mammals, with two variants expressed in humans (Fig. S1 A). The shorter human transcript (NM_001040025.3), encoding a 173 residue protein, is common among mammals, though a longer human isoform of 197 residues is also reported (NM_001040025.2). The longer variant corresponds to an mRNA starting from an upstream transcription initiation site, extending the 5' end to include an alternative upstream initiation codon. In mice, a frame-shifting indel precludes a similar extension of the coding sequence of the ARL16 gene (not shown), thus mice express only the 173 residue protein. The prevalent, shorter form results in a truncated N-terminus, compared to every other ARF family member, with the G-1 motif (GXXXGKT) beginning at residue 6, in contrast to residue 24 in ARF1 (Fig. S1 A). We predict this difference in the N-terminus has potentially important functional consequences for two reasons. First, the $\mathrm{N}$-termini of ARF family GTPases make direct contacts with binding partners/effectors, effectively serving as a conformationally sensitive "switch III" (Zhang et al., 2009). Also, the shorter form has a cysteine at residue 2 that is predicted to be S-palmitoylated (e.g., by the online prediction tool CSS-Palm (http://csspalm.biocuckoo.org/online.php)). In contrast, the $\mathrm{N}$-terminal extension in the longer isoform moves the cysteine well into the protein where it is no longer predicted to be palmitoylated.

ARL16 proteins retain most of the "G-motifs" (highlighted in red in Fig. S1 A and boxed in red in Fig. S1 B) present in ARF and RAS superfamily members. Mammalian ARL16 retained the conserved PTXG (G-2) motif that is critical in interconversion between the active and inactive conformations as well as the consensus G-4 motif (NKXD) found in almost all nucleotide binding proteins. In contrast, the ARL16 G-3 motif is altered from the highly conserved WDXGGQ (in which the glutamine participates directly in GTP hydrolysis) to RELGGC in ARL16 from multiple species, including humans and mice (Fig. S1 A and B). This change suggests the use of an alternative mechanism of GTP hydrolysis and presents a challenge to researchers as it lacks the glutamine (Q71 in ARFs or Q61 in RAS) in this motif that is commonly mutated to generate a "dominant activating" mutant, used to identify novel functions when expressed in cells. 


\section{The phylogenetic profile of ARL16 predicts a cilium-associated function}

Using the database of ARF family GTPase sequences from 114 eukaryotic species described previously (Vargova et al., 2021), we examined the distribution pattern of GTPases missing from eukaryotes that lack ciliated (flagellated) cells or stages. This revealed a strong correlation between ciliated species and four ARF family members, ARL3, ARL6, ARL13, and ARL16 (Fig. 1). To further assess this emerging pattern, we exploited existing genome and transcriptome assemblies to check for the presence of ARL16 orthologs in 25 additional eukaryotes (Table S1, Table S2). The expansion of the sampling was guided by an attempt to cover representatives of additional major phylogenetic lineages of eukaryotes missing in the previous study and to include additional instances of independently evolved non-ciliated taxa. This expanded analysis further supported the view of ARL16 as a broadly conserved and ancient ARF family paralog, as virtually all major eukaryote lineages include at least some ARL16-carrying representative (Fig. 1; Table S1). Multiple alignment of the diverse eukaryote ARL16 sequences revealed that the N-terminal truncation characteristic of the shorter human variant (see above) is in fact a rule rather than exception among ARL16 orthologs (Fig. S1 B). The majority of ARL16 proteins across the eukaryote phylogeny are predicted to be S-palmitoylated near the Nterminus (Vargova et al., 2021), but many lack this modification as they do not have any cysteine residues in that region (Fig. S1 B). Interestingly, the G-4 motif, critical for the specificity of binding of guanine nucleotides (Wittinghofer and Vetter, 2011), is abrogated in ARL16 proteins from several unrelated taxa (Fig. S1), suggesting that these proteins may function as ATPases (Leipe et al., 2002). Overall, ARL16 appears to be a rapidly evolving ARF family subgroup with differences in its primary structure suggesting substantial variation in the molecular details of ARL16 functioning among taxa.

In contrast to some other ancestral ARF family paralogs, ARL16 does not appear to have undergone lineage-specific gene duplications as there are few paralogs with (potentially) differentiated function. The only two taxa with more than one ARL16 gene (the rotifer Adineta vaga and the parabasalid Trichomonas vaginalis, each have two paralogs) both accumulate duplicated genes due to whole-genome or massive segmental duplications, respectively (Barratt et al., 2016; Flot et al., 2013). Thus, the most conspicuous aspect of ARL16 evolution in eukaryotes is frequent independent loss. The distribution of ARL16 orthologs across the eukaryote tree implies $>30$ independent loss events, scattered across different time points (affecting recently evolved taxa as well as ancient radiation of major eukaryote taxa; Fig. 1). Notably, the expanded sampling confirmed the initial observation that ARL16 occurs only in eukaryotes for which the ability to form cilia is directly documented or at least predicted by genomic evidence (i.e., presence of genes encoding typical ciliary proteins; Fig. 1, Table S1). The loss of ARL16 in relation to the loss of the cilium is non-random with high statistical significance (best tail $p$ $>4 \times 10^{-6}$, pairwise comparisons as implemented in Mesquite package, see Materials and Methods). However, ARL16 is also absent from various taxa that retained a cilium (Fig. 1; Table S1). Based on these observations, we hypothesize that the cellular role of ARL16 is related to the cilium, more specifically to be dependent on it but not necessarily essential for the function of the cilium as such. To provide a context for the putative association of ARL16 and cilia, we investigated the distribution of ARL3, ARL6, and ARL13 orthologs in the same taxa as we did for ARL16. Like ARL16, orthologs of ARL3, ARL6, and ARL13 are virtually always absent from taxa that do not form cilia (the sole exception being two highly divergent putative ARL6 orthologs in the heterolobosean Neovahlkampfia damariscottae that may have been recruited for a novel cilium-independent function). Thus, ARL3, ARL6, and ARL13 can be missing from ciliated taxa but this occurs less frequently than for ARL16 (Fig. 1, Table S1). Altogether, these comparative genomic analyses provide a strong case for the hypothesis that ARL16 is functionally linked to the cilium.

\section{ARL16 localizes to primary cilia in cultured human RPE cells and photoreceptor cells of the human retina}

ARL proteins frequently are found in multiple locations in cells reflecting that a single ARL can perform multiple functions (Sztul et al., 2019). ARL16 was previously found in cytosol in HEK293 and HeLa cells (Yang et al., 2011). We performed immunofluorescence studies of endogenous ARL16 in MEFs and RPE1 cells. Despite the fact that the human and mouse ARL16 proteins (173 residue variant) share $86 \%$ identity, the only commercially available ARL 16 antibody is specific to the human protein. Thus, we could not identify specific staining of ARL16 in MEFs or in cryosections through murine retinae (data not shown). In contrast, staining of 
human retinal pigmented epithelial (hTert-RPE1 (RPE1)) cells revealed ARL16 localizes along the ciliary axoneme in a punctate manner (Fig. 2 A). ARL16 also localized to both the cytosol and mitochondria, as evidenced by its diffuse staining across the cell and co-localization with the mitochondrial protein HSP60, respectively (Fig. S2 A).

To further examine the localization of ARL16 in MEFs and RPE1 cells, we generated plasmids that direct human ARL16 or mouse ARL16-myc expression. We generated homologous plasmids for the long or short form of human ARL16. Upon expression of the 173 residue human or mouse proteins in MEFs, we observed diffuse ARL16 staining in the cytosol (Fig. S2 B). In contrast, the 197 residue human variant displayed little evidence of diffuse, cytosolic localization. Instead, tubular and punctate staining co-localized strongly with HSP60 at mitochondria, consistent with endogenous staining of RPE1 cells after methanol fixation (Fig. S2 A). Thus, different length human variants localize quite distinctly when exogenously expressed. Because the endogenous staining of ARL16 in RPE1 cells displays prominent mitochondrial localization, we believe that these cells express at least some of the longer form of ARL16. Despite the presence of endogenous ARL16 in cilia in RPE cells, we did not find evidence of the exogenous protein localizing to cilia in MEFs.

We also analyzed ARL16 localization in photoreceptor cells in the retina, a commonly studied ciliary model tissue. The light sensitive outer segment resembles a highly modified but well characterized primary cilium (May-Simera et al., 2017; Roepman and Wolfrum, 2007). We used indirect immunofluorescence staining on a human donor retina as previously reported (Davidson et al., 2013; Turn et al., 2021). Figure 2 B shows double labeling of ARL16 and centrin 3, a marker for the connecting cilium as well as the mother (basal body) and daughter centrioles of photoreceptor cells in human retinae. Prominent ARL16 immunofluorescence labelled the ciliary region (CR) and the inner segment of the photoreceptor layer. Other retinal layers, such as the outer and inner nuclear layer (ONL, INL), outer and inner plexiform layer (OPL, IPL) where retinal synapses are localized, and the ganglion cell layer (GCL) showed no substantial staining. Higher magnification of the ciliary region revealed the localization of ARL16 in the periciliary region at the base of the connecting cilium (stained by anti-centrin 3) in human photoreceptor cells (Fig. 2 B, lower panels). The centrin-3 co-staining further showed that ARL16 labeled the basal body next to the daughter centriole. The inner segment where all biosynthetic active organelles such as the Golgi and ER are found, also showed prominent ARL16 immunofluorescence. Similar localizations were previously found for ciliary molecules which participate in Golgi functions and/or Golgi and molecular transfer processes to ciliary base (Sedmak and Wolfrum, 2010). The localization at the base of the connecting cilium is in line with other GTPases of the ARF superfamily like ARL2 and the ARF GAP ELMOD2, which also localize to ciliary rootlets in the inner segment (Turn et al., 2021).

\section{CRISPR/Cas9 Knockout of Arl16 in MEFs}

To test the prediction of a role for ARL16 in ciliary function, and to assess broader questions of its function in cells, we used CRISPR/Cas9 to introduce frame-shifting mutations into the open reading frame of Arl16 in MEFs. These cells are frequently used in cell biological studies, easily imaged due to their flat morphology, commonly used in cilia research, and diploid (which facilitates genome editing analyses). We used two guides to target Cas9 to exon 2, just downstream of the ATG at the end of exon 1 in Arl16 and screened clonal lines for indels by DNA sequencing of targeted regions. We sought frameshifting mutations predicted to cause premature translation termination and loss of protein function. The lack of a mouse antibody precluded us from directly testing for absence of protein expression. Although there is strong evidence (Smits et al., 2019) that cells may express mutated proteins resulting from use of downstream start sites or alternative splicing, the small size of the Arl16 open reading frame and presence of multiple, essential G-motifs along the length of the protein makes expression of a functional protein after introduction of indels near the $\mathrm{N}$-terminus highly unlikely. Thus, we refer to these as knockout (KO) lines. We generated five Arl16 KO clonal lines from two different guides that we used in these studies (alleles are shown in Fig. S3 A). Because we only found minor differences between the KO lines, data are presented in aggregate. As a further test of specificity and to ensure against off-target effects influencing our phenotyping, we performed rescue experiments in which we exogenously expressed ARL16-myc in KO and WT lines and assessed the reversal of phenotypes arising from the KOs.

Because almost nothing is known about ARL16 functions, and to obtain an unbiased overview of the effect of ARL16 loss on cells, we screened markers of major organelles and the cytoskeleton to look for 
differences between the Arl16 KO lines and the parental WT MEFs. No obvious differences were observed in mitochondrial morphology or distribution (HSP60), f-actin (FITC-phalloidin), nuclear number, size, or morphology (Hoechst), or microtubule networks ( $\alpha / \beta$-tubulin) when cells were fixed and stained as indicated (Fig. S3 B). However, these screens revealed a number of cellular defects in Arl16 KO lines, which we describe below.

\section{Arl16 KO cells have reduced ciliation and increased ciliary length}

In light of the phylogenetic prediction of a role for ARL16 at cilia and its localization there, we examined Arl16 KO cilia. We used acetylated tubulin (Ac-Tu) staining throughout to mark the ciliary axoneme after inducing ciliation in cultured cells. All five of the Arl16 null lines displayed reductions in the percentage of ciliated cells, compared to WT MEFs (Fig. 3 A, B). When the data from the five KO lines were pooled, the KO's had about $50 \%$ as many ciliated cells as WT after $24 \mathrm{hrs}$. However, at later time points the differences between WT and $\mathrm{KO}$ lines decreased, down to $25 \%$ lower at $72 \mathrm{hrs}$ of serum starvation and without reaching statistical significance. Thus, there is a decrease in ciliation compared to WT.

To ensure that the changes in ciliation were due to the loss of ARL16 and not off-target effects, we expressed ARL16-myc in WT and Arl16 KO cells. One day after transfection, we serum starved for 24 hrs and scored the percentage ciliation in cells expressing ARL16-myc. Interestingly, the percentage of WT cells with cilia increased almost $20 \%$ upon ARL16-myc expression, though this difference did not reach statistical significance (Fig. $3 \mathrm{C}$; $\mathrm{p}>0.05$ ). Expression of ARL16-myc in Arl16 KO lines ( $\mathrm{n}=2$ lines) resulted in a larger increase in ciliation, to levels approaching, and not statistically different from, those seen in WT cells also expressing ARL16-myc. Thus, expression of ARL16-myc reversed the decrease in ciliation observed in Arl16 $\mathrm{KO}$ lines, supporting a role for ARL16 in ciliogenesis.

It was also evident that cilia in Arl16 KO cells are longer than those in WT cells. Using the CiliaQ Plugin in FIJI (Hansen et al., 2021), we measured cilia lengths after 48 hrs of serum starvation (Fig. 3 D, E). On average, cilia in Arl16 KO lines $(5.09 \mu \mathrm{m})$ are $~ 90 \%$ longer than in WT $(2.67 \mu \mathrm{m})$ cells, based upon measurement of AcTu staining.

To gain insights into likely causes of the deficits in ciliation in Arl16 KOs, we screened markers of ciliogenesis for differences compared to WT MEFs. CEP164 recruitment to mother centrioles is an early step in ciliogenesis and required for cilium formation, while CP110 uncapping is a later step just prior to axoneme elongation (Cajanek and Nigg, 2014; Yadav et al., 2016). We examined these proteins in WT and Arl16 KO MEFs and found no statistically significant differences in either CEP164 recruitment or CP110 uncapping after $24 \mathrm{hr}$ of serum starvation (Fig. S4 A, B). Thus, we conclude that the defect in ciliogenesis observed in the Arl16 KO MEFs is downstream of CP110 uncapping.

Ciliary rootlets surround the basal body, are important in centrosome cohesion, and often project a foot or extension that helps stabilize cilia (Hossain et al., 2020; Yang et al., 2005; Yang and Li, 2006; Yang et al., 2002). Thus, we stained WT and Arl16 KO cells for rootletin, the primary component of rootlets. The absence of ARL16 strongly correlated with rootlet fragmentation (Fig. S4 C, D). However, in contrast to results observed in Elmod2 KOs, we did not observe an increase in centrosome separation in Arl16 KOs, based upon staining for $\gamma$ tubulin to mark centrosomes (Fig. S4 E).

\section{Arl16 KO leads to loss of several proteins from cilia}

While cilia could be readily identified by Ac-Tu staining in all cells, ciliary ARL13B staining in Arl16 KOs was dramatically reduced compared to that seen in WT cells (Fig. 4 A, B). Because recruitment of ARL13B to cilia is required for ciliary accumulation of the other ciliary membrane proteins, specifically ARL3 and INPP5E (Gigante et al., 2020), we assessed the endogenous levels and localizations of each of these proteins to the cilia of Arl16 KO cells. We readily detected both ARL3 and INPP5E in cilia of control MEFs, but neither ARL3 nor INPP5E were found in Arl16 KO cilia (Fig. $4 \mathrm{C}-\mathrm{F}$ ). Finally, we assessed the localization of the ciliary transmembrane protein adenylyl cyclase 3 (AC3) and found that AC3 is also lost from cilia of Arl16 KOs.

To determine if there was a generalized disruption in ciliary protein traffic, or a more specific loss related to ARL13B and ARL3, we expressed GFP-tagged somatostatin receptor (SSTR3-GFP) and assessed its ciliary localization in WT and Arl16 KO MEFs. We observed similar SSTR3-GFP signal in the cilia of both Arl16 KOs and WT controls (Fig. S4 F). This suggests that import of certain proteins is unaltered in cells lacking ARL16, 
though results from the exogenous expression of a tagged protein carries with it known caveats. We also examined a protein marker of the transition zone, CEP290. In both WT and Arl6 KO cells, CEP290 localizes to the base of the cilium, indicating no evident defects in its localization upon ARL16 ablation (Fig. S4 G). Thus, there is selective loss of a subset of ciliary proteins in cells lacking ARL16.

\section{Shh signaling is defective in Arl16 KO cells}

In light of the loss of ARL13B from cilia in Arl16 KO lines, and its proven role in ciliary signaling, yet ability to signal from outside cilia, we investigated whether ARL16 regulates Sonic Hedgehog (Shh) signaling (Bay et al., 2018; Caspary et al., 2007; Gigante et al., 2020; Larkins et al., 2011). Treatment of MEFs with Shh causes changes in the ciliary protein content, as well as processing of Gli transcription factors that ultimately result in changes in nuclear transcription, including that of Ptch1 and Gli1 mRNAs (Li et al., 2021). Smoothened (SMO) dynamically localizes to the ciliary membrane in response to Shh stimulation, with SMO absent under control conditions and enriched upon stimulation with Shh ligand (Corbit et al., 2005). Cells were treated with Shh conditioned medium with serum starvation for $24 \mathrm{hr}$ and then stained for SMO. SMO robustly increases in WT cilia but fails to do so in Arl16 KOs (Fig. 5 A, B).

To monitor transcriptional changes resulting from treatment of MEFs with Shh ligand, we performed qPCR to measure Ptch1 and Gli1 mRNA levels (two well-known targets increased by Shh signaling) after Shh stimulation. We observed increases in the levels of each of these mRNAs in WT cells in response to Shh stimulation. In contrast, the responses were markedly blunted in Arl16 KO cells (Fig. 5 C). Thus, ARL16 is also required for two well characterized responses to Shh; SMO recruitment and transcriptional changes in target genes.

\section{IFT140 is absent from cilia of Arl16 KOs}

We also analyzed the localization of several IFT components in Arl16 KO cells. In WT cells, IFT140 is observed at the ciliary base and along the length of the cilium. In marked contrast, IFT140 associated with the cilium or base was strongly reduced in Arl16 KO lines (Fig. 6 A). IFT140 is one of three core subunits of the IFTA complex so its absence suggested the possibility of the absence of this entire complex. Surprisingly, when we examined another core IFT-A component, IFT144, we observed its presence in the cilium with no evidence of changes between WT and Arl16 KO cells (Fig. 6 B). While optimizing staining protocols for IFT140, we found that IFT140 also localizes to rootlets, displaying strong overlap with rootletin staining in both WT and Arl16 KO cells (Fig. S4 H), even after fragmentation of rootlets in the KO cells.

We also examined effects of Arl16 KO on core components of the IFT-B complex, which consists of two core complexes (B1-1 and B1-2) and a peripheral complex (Quidwai et al., 2021; Wang et al., 2021; Yang and Huang, 2019). IFT81 (B1-1) and IFT88 (B1-2) localize along the length of the cilium and are often enriched at the base and tip in MEFs (Quidwai et al., 2021). We observed no differences in the localization of either IFT88 or IFT81 in cilia of Arl16 KO cells (Fig. $6 \mathrm{C}$, D), with each of them localizing along the length of the cilium in both WT and KO cells. Therefore, we observed changes in the ciliary localization of a single IFT-A core component, IFT140, without apparent disruption of the other IFT-A or IFT-B components.

\section{IFT140 and INPP5E accumulate at the Golgi in Arl16 KO cells}

While we did not detect IFT140 in Arl16 KO cilia, we noted instead that IFT140 accumulated in an intracellular membranous structure, which we identified as the Golgi by co-staining with GM130 (Fig. 7 A). This Golgi staining of IFT140 was not evident in WT cells, and expression of ARL16-myc reversed the increased staining of IFT140 at Golgi (Fig. 7 A). The only other IFT protein previously known to be associated with the Golgi is IFT20 (Crouse et al., 2014; Follit et al., 2006; Keady et al., 2011). Consistent with these earlier results, we found that in WT cells, IFT20 localizes to the Golgi and overlaps strongly with GM130 staining. We stained for IFT20 in Arl16 KO cells and found no differences in its localization compared to WT cells (Fig. $7 \mathrm{~B}$ ). We examined the potential presence of other IFT-A (IFT144) or IFT-B (IFT88, IFT81) proteins at Golgi and no increases were evident. We then stained for several other Golgi markers (including $\beta$-COP, FAPP2, and GBF1) to assess whether overall Golgi structure may be altered in Arl16 KO cells. Each of these proteins localize to the Golgi in both WT and Arl16 KO cells, and we observed no differences in Golgi morphology or intensity of any of 
these markers, based on visual inspection (Fig. S3 B). Thus, while one component from each of the IFT-A and IFT-B complexes, IFT140 and IFT20, respectively, can be found at the Golgi, only IFT140 is increased in its abundance at the Golgi in Arl16 KOs, while IFT20 appears to be a resident Golgi protein in both WT and Arl16 KO MEFs.

Surprised by the accumulation of IFT140 in the Golgi in Arl16 KOs, we assessed the Golgi localization of the other proteins that were lost from cilia using a variety of staining protocols to see if any others were accumulating there. Staining of INPP5E at cilia is typically performed using PFA fixation and under these conditions we observed it in cilia of WT lines but not in Arl16 KO lines. However, after methanol fixation, we found INPP5E staining increased at the Golgi in Arl16 KO but not WT cells (Fig. 7 C). In contrast, neither ARL3 nor ARL13B were observed at the Golgi in either WT or Arl16 KO lines under any conditions examined.

\section{INPP5E but not IFT140 is lost from cilia and accumulates in the Golgi of Pde6d KOs}

The phosphatidylinositol phosphate (PIP) 5'-phosphatase INPP5E is farnesylated at its C-terminus. As a result, its traffic to cilia is thought to be dependent on the prenyl binding protein PDE6D (Cook et al., 2000; Fansa et al., 2016). The prevailing model postulates that PDE6D binds to INPP5E and carries it to the cilium where it is released by ARL3 and retained by ARL13B (Humbert et al., 2012; Kosling et al., 2018; Thomas et al., 2014) A high throughput, yeast two-hybrid screen found that in addition to binding ARL3, PDE6D also binds ARL16 (Rolland et al., 2014). Therefore, we generated Pde6d KO MEFs using the same protocols as those to generate Arl16 KOs (Fig. S5) to compare the phenotypes observed in the absence of Pde6d to those observed in the absence of Arl16. We generated 5 lines from two guide RNAs in which the Pde6d gene contained frameshifting mutations (alleles of lines used in these studies are shown in Fig. S5B).

We then characterized the Pde6d KO lines for cilia number and protein composition. We observed no changes in the percentage of ciliated cells or length of cilia between Pde6d KO and WT cells (Fig. 7 A,B). We also found no differences in the strength of staining of ARL13B or ARL3 in cilia of Pde6d KOs compared to WT cells (Fig. 8 C, D). In contrast, when we examined ciliary staining of INPP5E, it was absent from Pde6d and Arl16 $\mathrm{KO}$ lines while present in WT cells (Fig. 8 D). INPP5E was instead increased at the Golgi in Pde6d KO cells as in Arl16 KO cells (Fig. $7 \mathrm{G}$ ).

We asked next whether IFT140 is also altered in Pde6d KO cells. We found that IFT140 localizes in Pde6d KO cilia at comparable levels to WT cilia, and is not enriched at the Golgi (Fig. $8 \mathrm{~F}, \mathrm{H}$ ). Thus, while traffic of both IFT140 and INPP5E to cilia requires ARL16, only traffic of INPP5E appears to be dependent on PDE6D. These results are consistent with previous data showing PDE6D-dependence of INPP5E traffic to cilia, likely the result of the prenylated INPP5E cargo binding the PDE6D shuttle. Further, these data suggest that IFT140 and INPP5E traffic to cilia via different pathways, each of which is dependent upon, or regulated by, ARL16.

\section{DISCUSSION}

We used a combination of phylogenetic analyses, genome editing of MEFs, and cell biological approaches to identify the cellular functions of one of the last uncharacterized members of the mammalian ARF family of regulatory GTPases, ARL16. We identified a strong correlation between the presence of cilia with the presence of ARL16 in genomes across the eukaryotic spectrum, leading us to propose cilia as a major site of action. Consistent with our phylogenetics-based model, we found ARL16 localizes to cilia and basal bodies in RPE and retinal photoreceptor cells, respectively. We generated Arl16 KO MEF lines to identify gross changes in cellular biology resulting from its loss and to experimentally test its predicted role in one or more aspects of cilia. We found that the loss of ARL16 results in a reduction in ciliation, due to changes in a step downstream of CP110 uncapping. We also observed an increase in average ciliary length and a large decrease in the presence of ARL13B, ARL3, INPP5E, and IFT140 in cilia. However, we believe that the most important function of ARL16 in ciliary biology may lie in its role in the regulation of traffic of ciliary proteins from the Golgi to cilia. We found two such pathways that are compromised when cells lack ARL16, one used by IFT140 and another by INPP5E. Deficiency of either IFT140 or INPP5E at cilia is expected to spawn an unknown number of downstream phenotypes, as they are key regulators of traffic along cilia, of protein export (IFT140) and in the control of ciliary membrane phospholipid composition (INPP5E). We also provide data that support a model for ARL16 acting 
from multiple sites in cells, including basal bodies and Golgi. Thus, our findings highlight both the importance of ARL16 to multiple processes and sites (summarized in the model shown in Fig. 9) and also the challenges involved in future work aimed at identifying molecular mechanisms for each of its actions, as might be expected for such an ancient and highly conserved GTPase.

\section{Phylogenetic analyses predict role(s) for ARL16 in ciliary biology}

Our phylogenetic analyses demonstrated that $A R L 16$ is restricted to ciliated taxa, similar to the three ARL GTPases previously shown to have ciliary-related roles: ARL3, ARL6, and ARL13. The phylogenetic profile (or phyletic pattern) of a gene can be an extremely informative resource providing evolutionary as well as functional insights. Genes encoding proteins that underpin the cilium are a particularly good illustration of this concept. Numerous genes subsequently demonstrated to encode proteins representing constituents of cilia or being critical for its biogenesis had been originally predicted as candidate "ciliary genes" based on searching for genes whose phylogenetic distribution correlates with the ability of the taxa to form cilia (Avidor-Reiss et al., 2004; Li et al., 2004; Merchant et al., 2007; Nevers et al., 2017). Other ciliary genes were discovered by ad hoc observations, as is the case of the Ras superfamily protein RJL proposed to have a cilium-related role based on its phylogenetic distribution more than a decade ago (Elias and Archibald, 2009) and confirmed as a regulator of ciliogenesis recently (Piette et al., 2021).

ARL16 has been missed by most, if not all, previous comparative genomic screens for candidate ciliary taxa. One reason for this may stem from the high sequence divergence between ARL 16 orthologs from distantly related eukaryotes, which might have resulted in the failure of commonly used, automated orthology prediction tools to recognize ARL16 genes in different species. Another confounding factor may be that the ARL16-cilium correlation is less conspicuous than is the case of many other ciliary genes in that it is missing in some ciliated species. That not all ciliary genes exhibit the same distribution pattern neatly correlating with the cilium is not unusual, as this cellular structure is highly malleable across species (Moran et al., 2014). Perhaps most obvious is the difference between motile and primary cilia as the latter (analyzed in this study) lack a central pair of microtubules, dynein arms and radial spokes. This phenomenon is documented also by our phylogenetically broad survey of the distribution of ARL3, ARL6, and ARL13 (Fig. 1; Table S1). None is present in all ciliumbuilding species, with ARL3 being the least frequently missing. Hence, the absence of ARL16 from various ciliated eukaryotes by itself does not undermine the prediction that this GTPase has a specific cilium-associated role. In fact, the restriction of $A R L 3, A R L 6, A R L 13$ and $A R L 16$ to cilium-building eukaryotes can be interpreted as evidence that the cilium-associated role of these proteins, established by studying a limited set of model organisms, is a general property across eukaryotes as a whole. A prediction we can draw then is that the potential non-ciliary roles of these proteins are limited, making them dispensable whenever the cilium is lost. By extension, if $A R L 16$ acts to regulate traffic from the Golgi, it may do so specifically in a Golgi-cilia pathway or for a subset of proteins destined for the cilium.

\section{Deletion of Arl16 causes a delay and overall decrease in ciliation yet increased ciliary length}

We found that the percentage of cells that form cilia in response to serum starvation is reduced in Arl16 KOs and that the affected step of ciliogenesis is downstream of CP110 uncapping of the basal body. It is by no means a complete defect in ciliation in the absence of ARL16. At later timepoints after serum starvation, Arl16 KO cells achieve ciliation rates approaching WT MEFs, and by 72 hrs show no statistically significant differences from WT. More detailed tracking of centrioles and ciliation would be required to determine the precise step of ciliogenesis that is affected.

In addition, the cilia that do form in Arl16 KOs are on average 90\% longer than WT cilia (Fig. 3). While many things are known to result in shorter than average cilia (including the absence of ARL13B (Caspary et al., 2007), fewer things are known that increase ciliary length (Duran et al., 2016; Wang et al., 2021). One such regulator is Gli2-mediated autophagy of Ofd1 (Hsiao et al., 2018), though we did not explore this pathway in the Arl16 KOs.

We recently discovered that ciliary rootlets are linked to ciliogenesis and the ARF GTPase activating protein (GAP) ELMOD2 (Turn et al., 2021). Elmod2 KO resulted in rootlet fragmentation and increased centrosomal separation, each of which was reversed upon expression of fast cycling ARL2 (Turn et al., 2021). 
However, while the loss of ARL16 results in rootlet fragmentation, there is no difference in centrosome separation between Arl16 KO and WT cells. These somewhat surprising results both suggest a role for ARL16 in rootlet integrity and may also raise questions about the role of rootlets in centrosome cohesion.

\section{$A R L 16$ is required for localization of a subset of ciliary proteins}

We found that ARL16 modulates multiple aspects of cilia. Arl16 KO results in the marked reduction of several ciliary proteins including ARL13B, ARL3, and INPP5E. It is possible that the loss of some of these is a cascade effect beginning with the loss of $A R L 13 B$, as it has been shown to play important roles in binding and retention of both ARL3 and INPP5E in cilia (Gigante et al., 2020; Ismail et al., 2012; Kobayashi et al., 2009; Mourão et al., 2014; Schrick et al., 2006; Wiens et al., 2010). Farnesylated INPP5E is transported to the cilium by the carrier PDE6D where it is released by ARL3 (Fansa et al., 2016; Stephen et al., 2017). Therefore, the loss of INPP5E from cilia in Arl16 KOs may result directly from the loss of ARL3 and/or ARL13B. We also found a reduction of AC3 in Arl16 KO cilia, perhaps a result of altered ciliary PIP content due to the loss of INPP5E. Finally, changes in SMO recruitment (leading to defective Hh signaling) is likely due to the changes of these other ciliary proteins, each of which have been shown to regulate Hh signaling (Huangfu and Anderson, 2006; Humbert et al., 2012; Placzek and Briscoe, 2018).

\section{Golgi-cilia traffic is compromised by the lack of $A R L 16$}

The disruption of ciliary proteins often is accompanied by defects at the Golgi (Dafinger et al., 2011; Goncalves et al., 2010), and changes to the Golgi can affect ciliogenesis (Greer et al., 2014; Hurtado et al., 2011). While a few ciliary proteins also localize to the Golgi, (Baron Gaillard et al., 2011; Follit et al., 2006) the Golgi is close to the cilium both during ciliogenesis and after cilium maturation, suggesting that there is likely continuous transfer between these compartments (Evans et al., 2010; Poole et al., 1997). Some exclusive pathways transport specific cargos from the Golgi to the cilium, including rhodopsin and PKD2 (Kim et al., 2014; Ward et al., 2011). Furthermore, recent work has highlighted a Golgi to cilium traffic pathway for ciliary membrane proteins that is regulated by IFT-A (Quidwai et al., 2021).

IFT machinery is required for proper assembly and maintenance of primary cilia (Kannabiran, 2020; Lechtreck, 2015; Nachury, 2018; Roepman and Wolfrum, 2007). This machinery consists of two complexes; IFT$\mathrm{A}$, which is primarily responsible for retrograde transport from the cilium tip to the cell body, and IFT-B, which is critical for cilium assembly and anterograde transport. However, there is also mounting evidence of a role for IFT-A in anterograde transport as well (e.g., (Mukhopadhyay et al., 2010)). Both IFT-A and -B are multi-subunit complexes, with IFT-A containing 6 members (IFT140/144/139/122/121, and 43) and IFT-B further divided into two subcomplexes, a 10 subunit core (IFT-88/81/74/70/56/52/46/27/25, and 22), and a 6 subunit peripheral complex (IFT-172/80/57/54/38, and 20) (Jordan and Pigino, 2021). Little is known about where or how these complexes assemble in the cell prior to associating with cilia.

In Arl16 KO cells, we found that the IFT-A core component IFT140 is lost from the cilium and accumulates at the Golgi. To date, the only other IFT protein known to localize to the Golgi is IFT20 (Follit et al., 2006), which is anchored there by binding to GMAP210 (Follit et al., 2008) and is required for opsin traffic from the Golgi to cilia (Keady et al., 2011). Loss of either IFT20 or IFT140 causes similar degenerative phenotypes in both the cilium and retinal photoreceptor cells, and loss of either abrogates ciliogenesis, though phenotypes are more severe in the IFT20 deletion (Crouse et al., 2014). However, several IFT subunits are associated with vesicular traffic functions in both ciliated and non-ciliated cells (Yang and Huang, 2019). Interestingly, our data do not show changes in other examined IFT-A or -B components, leading us to conclude that IFT140 accumulation at Golgi is independent of the other IFT components and that IFT140 may be involved in a novel Golgi to cilium traffic pathway, or perhaps traffic to a distinct compartment at which assembly of the IFT-A complex takes place.

In addition to IFT140, INPP5E accumulates at the Golgi of Arl16 KOs. Traffic of INPP5E has been extensively studied as mutations in it are associated with both Joubert and MORM ciliopathies. Our data here build upon that model and add to it. We show that in both Arl16 KOs and Pde6d KOs, INPP5E accumulates at the Golgi. High throughput screens also identified PDE6D as an interactor of ARL16 (Luck et al., 2020; Rolland et al., 2014). Therefore, we hypothesize that PDE6D picks up INPP5E at the Golgi to carry it to cilia in an ARL16dependent manner. 


\section{Summary}

This initial analysis of ARL16 identified roles in Golgi-cilia traffic that are likely linked with its effects on the control of ciliary length and ciliation itself, though acting at distinct sites. Clearly, substantially more work is required to identify each of the sites and mechanisms by which ARL16 acts. The current study used mammalian cell culture and CRISPR/Cas9 introduced mutations as a model system, though the presence of ARL16 in almost all ciliated eukaryotes should provide alternative systems that are predicted to yield additional insights into ARL16 actions. Despite our survey of a limited number of ciliary proteins we identified a number of proteins whose presence in cilia is compromised by the loss of ARL16. These proteins have been shown previously to play important roles in Shh signaling (ARL13B, ARL3, INPP5E), axoneme integrity (ARL13B), phospholipid metabolism (INPP5E), and retrograde intraflagellar traffic (IFT140) acting at or in cilia. Our findings of INPP5E and IFT140 accumulation at the Golgi in Arl16 KOs and INPP5E but not IFT140 at the Golgi in Pde6d KOs, support our model of ARL16 regulating two novel, independent traffic pathways from the Golgi to cilia. Finally, in a parallel study carried out in our lab we found phenotypes very similar to those reported here in Arl16 KO lines when MEFs were deleted for either of the ARF/ARL GAPs ELMOD1 or ELMOD3 (Turn et al., 2021). In addition, expression of ARL16-myc in Elmod1 or Elmod3 KO cells was able to reverse those phenotypes, providing strong support for a model that includes these two proteins as acting in concert with ARL16 at the Golgi and in ciliary biology.

\section{MATERIALS AND METHODS}

\section{Phylogenetic profiling of ARL3, ARL6, ARL13, and ARL16}

The set of 114 eukaryotes previously analyzed for the composition of the ARF family gene complement (Vargova et al., 2021)_was expanded by 25 additional eukaryotes (see Table S1), selected based upon the following criteria: (1) representatives of those major eukaryote lineages absent from the previous study (for the first time analyzing members of Hemimastigophora, Telonemia, Alveida, Rhodelphidia, and the CRUMs supergroup); (2) members of previously unsampled lineages known to have independently lost the cilium (Microsporidia, Myxozoa, Zygnematophyceae, Euglyphida); and (3) cilium-bearing close relatives of ciliumlacking taxa (e.g., the flagellated filasterean Pigoraptor chileana in contrast to the previously analyzed nonciliated filasterean Capsaspora owczarzaki). Available genomic or transcriptomic data from these eukaryotes (sources provided in Table S2) were analyzed with BLAST searches (Altschul et al., 1997) to identify putative orthologs of ARL3, ARL6, ARL13, and ARL16. Sets of protein sequences predicted based on genomic assemblies and transcriptome assembly contigs were searched with BLASTP and TBLASTN, respectively, using an ARF1 sequence as a query. Because ARL16 is rather distant from other ARF family members and is not always identified with ARF1 as the probing query, we performed parallel analyses starting with a reference $A R L 16$ sequence as the BLAST query. Hits with the e-value $\leq 0.1$ were retrieved and blasted (with BLASTP and BLASTX in the case of protein and nucleotide sequences, respectively) against an in-house database of Ras superfamily protein sequences including the curated set of ARF family sequences reported previously (Vargova et al., 2021). Queries with the best 20 hits all corresponding to one of the four GTPases of interest (ARL3, ARL6, $A R L 13$, and $A R L 16)$ were assigned as orthologs of the respective ARL protein, unless recognized as obvious contaminants from a different taxon (an issue sometimes happening in the case of transcriptome assemblies). Very rarely particular queries retrieved a set of 20 best hits consisting of a mixture of various ARF family members including some of the four focal GTPases. In all such cases the respective queries apparently corresponded to highly divergent genes with low sequence similarity to the canonical ARF family members, and were hence discarded. We cannot exclude the possibility that some of these genes as well as some of the unclassified ARF family members from the previous analysis (Vargova et al., 2021) are evolutionarily derived from ARL3, ARL6, ARL13, or ARL16 (i.e., being their orthologs or lineage-specific paralogs). However, in such cases their sequence divergence presumably entails a functional shift compared to canonical representatives of the four ARL paralogs, so failing to recognize their ultimate origin is unlikely to significantly impact the functional inferences from the phylogenetic distribution pattern of the four focal ARF family proteins. To avoid the possibility that any of the four GTPases is scored as missing for the given newly analyzed species only because of an 
accidental absence of the respective protein sequence resulting from inherently inaccurate genome annotation, we checked directly the genome assemblies with targeted TBLASTN searches and queries representing the ARL type not found in predicted protein sequence set. All newly identified ARL3, ARL6, ARL13, and ARL16 sequences were manually checked and if needed, the respective gene models were corrected to obtain accurate and complete protein sequences (the corrections are provided in Table S2).

The distribution pattern of ARL3, ARL6, ARL13, and ARL16 in the total set of 139 eukaryotes was correlated with the presence of cilia. Each species was scored as ciliated or non-ciliated (Fig. 1; Table S1) based on literature searches. A few apparently non-ciliated taxa (the foraminiferan Reticulomyxa filosa and the green algae Ostreococcus lucimarinus and Coccomyxa subelipsoidea) possess subsets of genes encoding characteristic ciliary proteins, suggesting that these species may form cilia at unknown life stages or lost the ability to build these structures only recently (Glöckner et al., 2014; Li et al., 2020). The cilia in these species, if indeed formed, are presumably highly reduced, owing to the lack of genes encoding many core ciliary proteins. Consistent with this, these species either completely lack ARL3, ARL6, ARL13, and ARL16 or, in the case of $R$. filosa, possess only one of these ARLs (ARL6) together with an apparent pseudogene corresponding to ARL3; Table S1). To account for the uncertain status of the cilium in these taxa, its presence was coded as ambiguous for the subsequent comparative analyses. The lack of evidence for the presence of ciliated stages in some poorly studied amoeboid protists (Rigifila ramosa, Armaparvus languidus) without genome sequence data should not be interpreted as evidence of absence of the cilium in these organisms, but the consistent lack of all four ciliumassociated ARLs from the transcriptome data generated for them is consistent with the idea that all four, including ARL16, are not required when no cilium is formed. Hence, for the purpose of our correlation analysis, these species were scored as non-ciliated. Finally, the pelagophyte alga Aureococcus anophagefferens was not reported to have cilia, but it possesses three out of the four cilium-associated ARLs (Table S1) and was previously shown to encode various other ciliary proteins, so it likely forms a flagellated stage, most likely zoospores, like its relatives (for further details see Eliáš et al. (Eliáš et al., 2016)). Hence, we scored it as a ciliated eukaryote.

The strength of the dependence of the presence of ARL16 on the presence of the cilium was formally tested using the pairwise comparisons algorithm (Maddison, 2000) implemented in the Mesquite package (Maddison, W. P. and D.R. Maddison. 2021. Mesquite: a modular system for evolutionary analysis. Version 3.70. http://www.mesquiteproject.org). A strictly bifurcating tree representing the phylogenetic relationships of the 139 taxa analyzed was constructed on the basis of previously published molecular phylogenetic and phylogenomic analyses. The somewhat contentious branching order of the main eukaryote lineages was arbitrarily defined following the most recent and comprehensive phylogenomic study (Tice et al., 2021), but alternative topologies at the base of the eukaryote tree suggested by other studies would not impact the result of the correlation analysis, as they do not change the inferred positions of cilium and ARL16 loss events. The tree was loaded into Mesquite and a two-character matrix was built following the character coding presented in Table S1, with the character 1 corresponding to the cilium (three states: present, absent, and ambiguous) and the character 2 representing the presence or absence of ARL16. The Pairwise Comparison test was carried out with the "Most Pairs" option and 1000 pairings, and which gave the best tail $p$ value range from $1.2 \times 10^{-7}$ to $3.81 \times 10^{-6}$ across all the pairings.

\section{Reagents, Antibodies, and Plasmids}

All chemicals used were purchased from commercial sources. The following antibodies were used in these studies: ARL16 (1:100; Sigma; HPA043711), Acetylated Tubulin (1:1000; Sigma; T5192), Centrin clone $20 \mathrm{H} 5$ (1:1000; Sigma; 04-1624), polyclonal rabbit anti-centrin 3 (1:100 (Trojan et al., 2008)), myc (1:1000; Abcam; ab9132), HSP60 (1:1000; Stressgen; ADI-SPA-807), GM130 (1:1000; BD/Transduction; 610823), Tubulin (1:1000; EMD Millipore; MAB1864), $\beta$-COP (1:2,000; ThermoFisher PA1-061), FAPP2 (Gift from Antonella De Matteis), GBF1 (1:200; BD; 612116), CP110 (1:100; Proteintech; 66448-1-ig), gamma tubulin (1:1000; Sigma T6557 or Abcam ab11317), CEP164 (1:100; Santa Cruz; sc-515403), rootletin (1:1000; EMD Millipore; ABN1686), CEP290 (1:100; Proteintech; 22490-1-ap), NPHP4 (1:100; Proteintech; 13812-1-ap), IFT81(1:200; Proteintech; 11744-1AP), ARL13B (1:500; Proteintech; 17711-1-AP), ARL3(1:100; R75448 (Cavenagh et al., 1994), INPP5E (1:100; Proteintech; 17797-1-ap), AC3 (1:100; LSBio; LS-C204505/183274), 
SMO (gift from Kathryn Anderson (Ocbina et al., 2011), GLI3 (1:1000; R\&D Systems; AF3690), IFT140 (1:200; Proteintech; 17460-1-AP), IFT144(1:100; Proteintech; 13647-1-AP), IFT88(1:200; Proteintech; 13967-1-ap), and IFT20(gift from Greg Pazour (Pazour et al., 2002)).

The plasmid directing expression of mouse ARL16-myc in mammalian cells was obtained by first having the open reading frame synthesized by GenScript and later using PCR to amplify this open reading frame with insertion of the C-terminal myc epitope (EQKLISEEDL) after a diglycine linker. The PCR product was ligated into pCDNA3.1 using Kpnl and Xhol sites and the entire open reading frame was sequenced to confirm fidelity. The plasmid expressing SSTR3-GFP was a gift from Max Nachury (Marley et al., 2013).

\section{Cell culture}

All cells were maintained in DMEM (ThermoFisher \#11965) supplemented with $10 \% \mathrm{FBS}$ at $37^{\circ} \mathrm{C}$ and $5 \% \mathrm{CO}_{2}$. To induce ciliation, media was swapped with DMEM supplemented with $0.5 \%$ FBS for $48 \mathrm{~h}$ unless otherwise indicated. Cells were grown in the absence of antibiotics. Routine screening for mycoplasma contamination was performed using DNA staining.

\section{CRISPR/Cas9}

Genes of interest were disrupted in immortalized WT MEFs (ATCC CRL-2991) with CRISPR/Cas9 gene editing, as previously described (Schiavon et al., 2019; Turn et al., 2020; Turn et al., 2021). Guide RNA sequences targeting the coding region of the gene were designed using Benchling (benchling.com/academic). For Arl16 the guides used were Guide 2: GGAGAGCCCCCACCGACGCGG and Guide 3: CGGAGATGGCAAAGGCGACCT. For Pde6d the guides used were Guide 1: GCAAATGGAAAAATTCCGCC and Guide 3: CACCGCCTTCGGGATGCCGAAACA.

Double stranded oligonucleotides of the guide sequences (with a substitution of a G for the first nucleotide to facilitate expression from the U6 promotor) were cloned into the pSpCas9(BB)-2A-Puro (PX459) V2.0 Vector (Addgene) at the Bbsl sites. Cells were transfected with the resulting plasmid with a 1:3 ratio of DNA (2 $\mu \mathrm{g})$ to Lipofectamine $2000(6 \mu \mathrm{g})$ for $4 \mathrm{hrs}$ in OptiMEM, according to manufacturer's instructions. Cells were then replated and allowed to recover overnight in DMEM with $10 \%$ FBS. Cells were then grown in $3 \mu \mathrm{g} / \mathrm{ml}$ puromycin for 4 days, to enrich for transfected cells. Cells were then seeded into two 96 -well plate at densities of 3-5 cells/well and monitored visually during growth. Clones resulting from single cells were isolated, expanded, and cryopreserved. PCR primers were designed to amplify genomic DNA outside the target site to allow sequencing of genomic DNA to identify and verify frameshifting mutations. Note that clones harboring no changes in the targeted region were often retained and referred to as "CRISPR WT" cells as they had been through the transfection, selection, and cloning process as the null lines and serve as another control against off target effects.

\section{Transfection}

Cells were transfected with $4 \mu \mathrm{g}$ DNA: $4 \mu \mathrm{L}$ JetOPTIMUS transfection reagent (VWR, 76299-634) according to manufacturer guidelines in standard medium overnight. The next day, cells were replated on coverslips in standard medium and allowed to recover for $24 \mathrm{hr}$. Cells were then serum starved for 24-72 hr to induce ciliation prior to fixation.

\section{Immunofluorescence}

All cells were cultured on coverslips coated with matrigel (BD Biosciences \#356231).

PFA protocol: For the following antibodies (Ac-Tu, ARL13B, ARL3, INPP5E, $\gamma$-tubulin, SMO, AC3), cells were fixed $4 \%$ paraformaldehyde (PFA) in PBS pre-warmed to $37^{\circ} \mathrm{C}$ on the benchtop for 15 min. Cells were permeabilized with $0.1 \%$ Triton-X 100 in PBS for 10 minutes at room temperature. For Ac-Tu, ARL13B, and SMO, cells were blocked with 1\% bovine serum albumin (Sigma \#A3059) in PBS for 1 hr. Primary antibodies were diluted in blocking solution and applied to cells at $4^{\circ} \mathrm{C}$ overnight. Cells were washed $4 \times 5$ min with PBS before incubating with secondary antibodies (1:1000 Alexa fluorophores, ThermoFisher) in blocking solution for $1 \mathrm{hr}$ at room temperature. Cells were washed $4 \times 5$ min with PBS before being mounted onto slides with MOWIOL. For ARL3, INPP5E, and AC3, a blocking solution of $10 \%$ FBS in PBS was used in place of $1 \%$ BSA. 
Methanol protocol: For these antibodies ( $\gamma$-tubulin, Ac-Tu, INPP5E at Golgi, CEP164, and CEP290), cells were fixed with methanol at $-20^{\circ} \mathrm{C}$ for $10 \mathrm{~min}$, washed in PBS with agitation. Cells were blocked with blocking buffer (PBS with 10\% FBS) for 30 min at room temperature. Primary antibodies were diluted in blocking buffer and applied to the cells at $4^{\circ} \mathrm{C}$ overnight. Cells were washed $3 \times 5 \mathrm{~min}$ with PBS with agitation before applying secondary antibody diluted 1:500 in blocking buffer for $1 \mathrm{hr}$ at room temperature. Cells were then washed and mounted as described above.

IFT protocol: For IFT staining (IFT140, IFT144, IFT88, and IFT81). Immediately out of the incubator, cells were washed $2 \times$ in PBS warmed to $37^{\circ} \mathrm{C}$ prior to fixation with 4\% PFA in PHEM (60 mM PIPES, 22 mM HEPES, $10 \mathrm{mM}$ EGTA, $4 \mathrm{mM} \mathrm{MgSO} 4-7 \mathrm{H}_{2} \mathrm{O}, \mathrm{pH}$ 6.9) for $15 \mathrm{~min}$ at room temperature. Cells were washed $2 x$ with PBS and treated with $50 \mathrm{mM} \mathrm{NH}_{4} \mathrm{Cl}$ twice for $15 \mathrm{~min}$. Cells were washed again with PBS before permeabilization with $0.25 \%$ Triton-X100 in PBS for 10 min at room temperature. Cells were blocked with $10 \%$ FBS in PBS with $0.2 \%$ Tween-20 for 60 min at room temperature and then incubated with primary antibodies in $1 \%$ FBS in PBS with $0.025 \%$ Triton-X100 at room temp for $1 \mathrm{hr}$ or at $4^{\circ} \mathrm{C}$ overnight. Cells were washed $4 \times 10$ min with PBS before incubation with secondary antibodies for $60 \mathrm{~min}$ at room temp. Cells were rinsed with $0.25 \%$ Triton-X100 in PBS $5 \times 10$ min before mounting as above.

\section{Microscopy}

Samples were visualized using an Olympus IX81 widefield microscope and Slidebook software; 100X magnification [UPlanFI, 1.30 NA Oil]. Images were processed and analyzed using FIJI image analysis software. Any images appearing in the same panel of a figure were processed identically including objectives, acquisition settings, cropping, brightness adjustments, and any other processing settings.

\section{Scoring of cell phenotypes}

For all phenotypes that were scored, experiments were performed in triplicate and scored at least in duplicate as indicated in the corresponding figure legends. For percent ciliated cells, cilia were identified using Ac-Tu. For presence of markers in cilia (ARL13B, ARL3, INPP5E, etc.), we binned them as either present (visible even without checking the Ac-Tu channel), reduced (present, but only noticeable upon switching to Ac-Tu channel), or absent (cannot be detected upon switching to Ac-Tu channel). For all ciliary phenotyping, Ac-Tu was used to define cilia. For centrosomal/basal body scoring, $\gamma$-tubulin was used as the standard comparison point. Finally, for Golgi staining/localization, GM130 was used to define the Golgi.

\section{Human Tissue}

The human donor eye tissue applied in the present study was obtained $11.5 \mathrm{hr}$ post mortem from a female donor (\# 252-09), 65 years of age without any underlying health conditions, from the Department of Ophthalmology, University Medical Center Mainz, Germany. The guidelines to the declaration of Helsinki (http://www.wma.net/en/30publications/ 10policies/b3/) were followed.

\section{Immunohistochemistry of Retinal Sections}

Human retinae were dissected from enucleated eye balls and cryofixed in melting isopentane and cryosectioned at $-20^{\circ} \mathrm{C}$ in a cryostat (HM 560 Cryo-Star; MICROM) as previously described (Karlstetter et al., 2014; Wolfrum, 1991). $10 \mu \mathrm{m}$ sections were placed on poly-L-lysine-precoated coverslips and incubated with $0.01 \%$ Tween 20 in PBS for 20 min. After washing, sections were flooded with blocking solution $(0.5 \%$ coldwater fish gelatin plus $0.1 \%$ ovalbumin in PBS) and incubated for at least 30 min followed by an overnight incubation with primary antibodies at $4^{\circ} \mathrm{C}$ in blocking solution (Trojan et al., 2008). Washed cryosections were incubated with secondary antibodies conjugated to Alexa 488 or Alexa 568 (Invitrogen) in blocking solution and with DAPI (Sigma-Aldrich) to stain the DNA of nuclei, for $1.5 \mathrm{~h}$ at room temperature in the dark. After three washes in PBS, specimens were mounted in Mowiol 4.88 (Hoechst) and imaged using a Leica DM6000B deconvolution microscope. 


\section{Shh assay}

Shh response was determined by measuring transcriptional changes in Gli1 and Ptch1 mRNA levels, as previously described (Mariani et al., 2016). Cells were maintained in low serum (0.5\% FBS) media for 48 hrs either with or without Shh conditioning. RNA was prepared using the Qiagen RNeasy Kit with QIAshredder homogenizer columns, according to the manufacturer's protocols. RNA (200 ng) was used to generate cDNAs using BioRad iScript Reverse Transcription supermix. The following primers were used for quantitative PCR (qPCR):

\section{Pold3 (housekeeping gene)}

\section{F: 5'-ACGCTTGACAGGAGGGGGCT-3' \\ R: 5'-AGGAGAAAAGCAGGGGCAAGCG-3'}

Gli1

F: 5'-CTTCACCCTGCCATGAAACT-3'

R: 5'-TCCAGCTGAGTGTTGTCCAG-3'

\section{Ptch1}

F: 5'-TGCTGTGCCTGTGGTCATCCTGATT-3'

R: 5'-CAGAGCGAGCATAGCCCTGTGGTTC-3'

In brief, the cDNA was combined with primers and Bio-Rad SsoAdvanced Universal SYBR Supermix according to the manufacturer's protocols (1725270). Samples were run on a Bio-Rad CFX96 Touch Real-Time PCR Detection System, and data were analyzed using Bio-Rad CFX Manager 3.1. The following program conditions were used: $95^{\circ} \mathrm{C}$ for $5 \mathrm{~min} ; 45$ cycles of $95^{\circ} \mathrm{C}$ for $15 \mathrm{~s} ; 57^{\circ} \mathrm{C}$ for $30 \mathrm{~s}$. Reactions were performed in technical duplicate on three biological replicates. Data were then analyzed by the $\Delta \Delta C T$ method and normalized to control WT levels for each transcriptional target (Livak and Schmittgen, 2001).

\section{Reproducibility and Statistics}

All data were plotted using GraphPad Prism. Error bars represent the standard error of the mean (SEM), and box-and-whisker plots indicate the range of the data along with the median and upper/lower quartiles. Ttests or one way ANOVA tests were used to determine whether there were significant differences between test groups as indicated. Asterisks in a figure indicates statistical significance: ${ }^{*} p<0.05,{ }^{* *} p<0.01,{ }^{* * *} p<0.001$. Actual $p$ values are indicated in the figure legends.

\section{ACKNOWLEDGEMENTS}

This work was supported by grants from the NIH (R35GM122568 to RAK, R35GM122549 to TC, and F31HD096815 to SID), ERD Funds, project OPVVV CZ.02.1.01/0.0/0.0/16_019/0000759 (Centre for research of pathogenicity and virulence of parasites), Czech Science Foundation grant 20-27648S and ERD Funds, project OPVVV CZ.02.1.01/0.0/0.0/16_019/0000759 (to M.E.). We also thank Mike Murphy, RefSeq Curator $(\mathrm{NCBI} / \mathrm{NLM} / \mathrm{NIH})$ for clarification on mRNA variants of the human ARL16 mRNA. The authors regret that due to the journal's limitation on words in the text we are unable to cite many important contributions in the primary literature.

\section{REFERENCES}


Altschul, S.F., T.L. Madden, A.A. Schaffer, J. Zhang, Z. Zhang, W. Miller, and D.J. Lipman. 1997. Gapped BLAST and PSI-BLAST: a new generation of protein database search programs. Nucleic Acids Res. 25:33893402.

Avidor-Reiss, T., A.M. Maer, E. Koundakjian, A. Polyanovsky, T. Keil, S. Subramaniam, and C.S. Zuker. 2004. Decoding cilia function: defining specialized genes required for compartmentalized cilia biogenesis. Cell. 117:527-539.

Baron Gaillard, C.L., E. Pallesi-Pocachard, D. Massey-Harroche, F. Richard, J.P. Arsanto, J.P. Chauvin, P. Lecine, H. Kramer, J.P. Borg, and A. Le Bivic. 2011. Hook2 is involved in the morphogenesis of the primary cilium. Mol Biol Cell. 22:4549-4562.

Barratt, J., R. Gough, D. Stark, and J. Ellis. 2016. Bulky Trichomonad Genomes: Encoding a Swiss Army Knife. Trends in parasitology. 32:783-797.

Bay, S.N., A.B. Long, and T. Caspary. 2018. Disruption of the ciliary GTPase Arl13b suppresses Sonic hedgehog overactivation and inhibits medulloblastoma formation. Proc Natl Acad Sci U S A. 115:1570-1575.

Breslow, D.K., S. Hoogendoorn, A.R. Kopp, D.W. Morgens, B.K. Vu, M.C. Kennedy, K. Han, A. Li, G.T. Hess, M.C. Bassik, J.K. Chen, and M.V. Nachury. 2018. A CRISPR-based screen for Hedgehog signaling provides insights into ciliary function and ciliopathies. Nat Genet. 50:460-471.

Cajanek, L., and E.A. Nigg. 2014. Cep164 triggers ciliogenesis by recruiting Tau tubulin kinase 2 to the mother centriole. Proc Natl Acad Sci U S A. 111:E2841-2850.

Carter, S.P., and O.E. Blacque. 2019. Membrane retrieval, recycling and release pathways that organise and sculpt the ciliary membrane. Curr Opin Cell Biol. 59:133-139.

Caspary, T., C.E. Larkins, and K.V. Anderson. 2007. The graded response to Sonic Hedgehog depends on cilia architecture. Dev Cell. 12:767-778.

Cavenagh, M.M., M. Breiner, A. Schurmann, A.G. Rosenwald, T. Terui, C. Zhang, P.A. Randazzo, M. Adams, H.G. Joost, and R.A. Kahn. 1994. ADP-ribosylation factor (ARF)-like 3, a new member of the ARF family of GTP-binding proteins cloned from human and rat tissues. J Biol Chem. 269:18937-18942.

Chiang, A.P., D. Nishimura, C. Searby, K. Elbedour, R. Carmi, A.L. Ferguson, J. Secrist, T. Braun, T. Casavant, E.M. Stone, and V.C. Sheffield. 2004. Comparative genomic analysis identifies an ADP-ribosylation factor-like gene as the cause of Bardet-Biedl syndrome (BBS3). Am J Hum Genet. 75:475-484.

Cook, T.A., F. Ghomashchi, M.H. Gelb, S.K. Florio, and J.A. Beavo. 2000. Binding of the delta subunit to rod phosphodiesterase catalytic subunits requires methylated, prenylated C-termini of the catalytic subunits. Biochemistry. 39:13516-13523.

Corbit, K.C., P. Aanstad, V. Singla, A.R. Norman, D.Y. Stainier, and J.F. Reiter. 2005. Vertebrate Smoothened functions at the primary cilium. Nature. 437:1018-1021.

Crouse, J.A., V.S. Lopes, J.T. Sanagustin, B.T. Keady, D.S. Williams, and G.J. Pazour. 2014. Distinct functions for IFT140 and IFT20 in opsin transport. Cytoskeleton (Hoboken). 71:302-310.

Dafinger, C., M.C. Liebau, S.M. Elsayed, Y. Hellenbroich, E. Boltshauser, G.C. Korenke, F. Fabretti, A.R. Janecke, I. Ebermann, G. Nurnberg, P. Nurnberg, H. Zentgraf, F. Koerber, K. Addicks, E. Elsobky, T. Benzing, B. Schermer, and H.J. Bolz. 2011. Mutations in KIF7 link Joubert syndrome with Sonic Hedgehog signaling and microtubule dynamics. J Clin Invest. 121:2662-2667.

Davidson, A.E., N. Schwarz, L. Zelinger, G. Stern-Schneider, A. Shoemark, B. Spitzbarth, M. Gross, U. Laxer, J. Sosna, P.I. Sergouniotis, N.H. Waseem, R. Wilson, R.A. Kahn, V. Plagnol, U. Wolfrum, E. Banin, A.J. Hardcastle, M.E. Cheetham, D. Sharon, and A.R. Webster. 2013. Mutations in ARL2BP, encoding ADPribosylation-factor-like 2 binding protein, cause autosomal-recessive retinitis pigmentosa. Am J Hum Genet. 93:321-329.

Derelle, R., G. Torruella, V. Klimes, H. Brinkmann, E. Kim, C. Vlcek, B.F. Lang, and M. Elias. 2015. Bacterial proteins pinpoint a single eukaryotic root. Proc Natl Acad Sci U S A. 112:E693-699.

Dishinger, J.F., H.L. Kee, P.M. Jenkins, S. Fan, T.W. Hurd, J.W. Hammond, Y.N. Truong, B. Margolis, J.R. Martens, and K.J. Verhey. 2010. Ciliary entry of the kinesin-2 motor KIF17 is regulated by importin-beta2 and RanGTP. Nat Cell Biol. 12:703-710. 
Duran, I., S.P. Taylor, W. Zhang, J. Martin, K.N. Forlenza, R.P. Spiro, D.A. Nickerson, M. Bamshad, D.H. Cohn, and D. Krakow. 2016. Destabilization of the IFT-B cilia core complex due to mutations in IFT81 causes a Spectrum of Short-Rib Polydactyly Syndrome. Sci Rep. 6:34232.

Elias, M., and J.M. Archibald. 2009. The RJL family of small GTPases is an ancient eukaryotic invention probably functionally associated with the flagellar apparatus. Gene. 442:63-72.

Eliáš, M., V. Klimeš, R. Derelle, R. Petrželková, and J. Tachezy. 2016. A paneukaryotic genomic analysis of the small GTPase RABL2 underscores the significance of recurrent gene loss in eukaryote evolution. Biology direct. 11:5.

Evans, R.J., N. Schwarz, K. Nagel-Wolfrum, U. Wolfrum, A.J. Hardcastle, and M.E. Cheetham. 2010. The retinitis pigmentosa protein RP2 links pericentriolar vesicle transport between the Golgi and the primary cilium. Human molecular genetics. 19:1358-1367.

Fan, Y., M.A. Esmail, S.J. Ansley, O.E. Blacque, K. Boroevich, A.J. Ross, S.J. Moore, J.L. Badano, H. MaySimera, D.S. Compton, J.S. Green, R.A. Lewis, M.M. van Haelst, P.S. Parfrey, D.L. Baillie, P.L. Beales, N. Katsanis, W.S. Davidson, and M.R. Leroux. 2004. Mutations in a member of the Ras superfamily of small GTP-binding proteins causes Bardet-Biedl syndrome. Nat Genet. 36:989-993.

Fansa, E.K., S.K. Kosling, E. Zent, A. Wittinghofer, and S. Ismail. 2016. PDE6delta-mediated sorting of INPP5E into the cilium is determined by cargo-carrier affinity. Nat Commun. 7:11366.

Fisher, S., D. Kuna, T. Caspary, R.A. Kahn, and E. Sztul. 2020. ARF family GTPases with links to cilia. Am J Physiol Cell Physiol. 319:C404-C418.

Flot, J.F., B. Hespeels, X. Li, B. Noel, I. Arkhipova, E.G. Danchin, A. Hejnol, B. Henrissat, R. Koszul, J.M. Aury, V. Barbe, R.M. Barthélémy, J. Bast, G.A. Bazykin, O. Chabrol, A. Couloux, M. Da Rocha, C. Da Silva, E. Gladyshev, P. Gouret, O. Hallatschek, B. Hecox-Lea, K. Labadie, B. Lejeune, O. Piskurek, J. Poulain, F. Rodriguez, J.F. Ryan, O.A. Vakhrusheva, E. Wajnberg, B. Wirth, I. Yushenova, M. Kellis, A.S. Kondrashov, D.B. Mark Welch, P. Pontarotti, J. Weissenbach, P. Wincker, O. Jaillon, and K. Van Doninck. 2013. Genomic evidence for ameiotic evolution in the bdelloid rotifer Adineta vaga. Nature. 500:453-457.

Follit, J.A., J.T. San Agustin, F. Xu, J.A. Jonassen, R. Samtani, C.W. Lo, and G.J. Pazour. 2008. The Golgin GMAP210/TRIP11 anchors IFT20 to the Golgi complex. PLoS genetics. 4:e1000315.

Follit, J.A., R.A. Tuft, K.E. Fogarty, and G.J. Pazour. 2006. The intraflagellar transport protein IFT20 is associated with the Golgi complex and is required for cilia assembly. Mol Biol Cell. 17:3781-3792.

Gigante, E.D., and T. Caspary. 2020. Signaling in the primary cilium through the lens of the Hedgehog pathway. Wiley Interdiscip Rev Dev Biol:e377.

Gigante, E.D., M.R. Taylor, A.A. Ivanova, R.A. Kahn, and T. Caspary. 2020. ARL13B regulates Sonic hedgehog signaling from outside primary cilia. Elife. 9.

Glöckner, G., N. Hülsmann, M. Schleicher, A.A. Noegel, L. Eichinger, C. Gallinger, J. Pawlowski, R. Sierra, U. Euteneuer, L. Pillet, A. Moustafa, M. Platzer, M. Groth, K. Szafranski, and M. Schliwa. 2014. The genome of the foraminiferan Reticulomyxa filosa. Current biology : CB. 24:11-18.

Goetz, S.C., P.J. Ocbina, and K.V. Anderson. 2009. The primary cilium as a Hedgehog signal transduction machine. Methods Cell Biol. 94:199-222.

Goncalves, J., S. Nolasco, R. Nascimento, M. Lopez Fanarraga, J.C. Zabala, and H. Soares. 2010. TBCCD1, a new centrosomal protein, is required for centrosome and Golgi apparatus positioning. EMBO Rep. 11:194-200.

Gotthardt, K., M. Lokaj, C. Koerner, N. Falk, A. GießI, and A. Wittinghofer. 2015. A G-protein activation cascade from Arl13B to Arl3 and implications for ciliary targeting of lipidated proteins. Elife. 4.

Greer, Y.E., C.J. Westlake, B. Gao, K. Bharti, Y. Shiba, C.P. Xavier, G.J. Pazour, Y. Yang, and J.S. Rubin. 2014. Casein kinase 1delta functions at the centrosome and Golgi to promote ciliogenesis. Mol Biol Cell. 25:1629-1640.

Hansen, J.N., S. Rassmann, B. Stüven, N. Jurisch-Yaksi, and D. Wachten. 2021. CiliaQ: a simple, open-source software for automated quantification of ciliary morphology and fluorescence in 2D, 3D, and 4D images. The European Physical Journal E. 44:18.

Hossain, D., S.Y.-P. Shih, X. Xiao, J. White, and W.Y. Tsang. 2020. Cep44 functions in centrosome cohesion by stabilizing rootletin. Journal of Cell Science. 133:jcs239616. 
Hsiao, C.J., C.H. Chang, R.B. Ibrahim, I.H. Lin, C.H. Wang, W.J. Wang, and J.W. Tsai. 2018. Gli2 modulates cell cycle re-entry through autophagy-mediated regulation of the length of primary cilia. $J$ Cell Sci. 131.

Huangfu, D., and K.V. Anderson. 2006. Signaling from Smo to Ci/Gli: conservation and divergence of Hedgehog pathways from Drosophila to vertebrates. Development. 133:3-14.

Humbert, M.C., K. Weihbrecht, C.C. Searby, Y. Li, R.M. Pope, V.C. Sheffield, and S. Seo. 2012. ARL13B, PDE6D, and CEP164 form a functional network for INPP5E ciliary targeting. Proc Natl Acad Sci U S A. 109:19691-19696.

Hurtado, L., C. Caballero, M.P. Gavilan, J. Cardenas, M. Bornens, and R.M. Rios. 2011. Disconnecting the Golgi ribbon from the centrosome prevents directional cell migration and ciliogenesis. The Journal of cell biology. 193:917-933.

Ismail, S.A., Y.X. Chen, M. Miertzschke, I.R. Vetter, C. Koerner, and A. Wittinghofer. 2012. Structural basis for Arl3-specific release of myristoylated ciliary cargo from UNC119. Embo j. 31:4085-4094.

Ivanova, A.A., T. Caspary, N.T. Seyfried, D.M. Duong, A.B. West, Z. Liu, and R.A. Kahn. 2017. Biochemical characterization of purified mammalian ARL13B protein indicates that it is an atypical GTPase and ARL3 guanine nucleotide exchange factor (GEF). J Biol Chem. 292:11091-11108.

Jacoby, M., J.J. Cox, S. Gayral, D.J. Hampshire, M. Ayub, M. Blockmans, E. Pernot, M.V. Kisseleva, P. Compère, S.N. Schiffmann, F. Gergely, J.H. Riley, D. Pérez-Morga, C.G. Woods, and S. Schurmans. 2009. INPP5E mutations cause primary cilium signaling defects, ciliary instability and ciliopathies in human and mouse. Nat Genet. 41:1027-1031.

Jordan, M.A., and G. Pigino. 2021. The structural basis of intraflagellar transport at a glance. Journal of Cell Science. 134.

Kannabiran, C. 2020. Review: Intraflagellar transport proteins in the retina. Mol Vis. 26:652-660.

Karlstetter, M., N. Sorusch, A. Caramoy, K. Dannhausen, A. Aslanidis, S. Fauser, M.R. Boesl, K. Nagel-Wolfrum, E.R. Tamm, H. Jagle, H. Stoehr, U. Wolfrum, and T. Langmann. 2014. Disruption of the retinitis pigmentosa 28 gene Fam161a in mice affects photoreceptor ciliary structure and leads to progressive retinal degeneration. Human molecular genetics. 23:5197-5210.

Keady, B.T., Y.Z. Le, and G.J. Pazour. 2011. IFT20 is required for opsin trafficking and photoreceptor outer segment development. Mol Biol Cell. 22:921-930.

Kim, H., H. Xu, Q. Yao, W. Li, Q. Huang, P. Outeda, V. Cebotaru, M. Chiaravalli, A. Boletta, K. Piontek, G.G. Germino, E.J. Weinman, T. Watnick, and F. Qian. 2014. Ciliary membrane proteins traffic through the Golgi via a Rabep1/GGA1/Arl3-dependent mechanism. Nat Commun. 5.

Kobayashi, T., Y. Hori, N. Ueda, H. Kajiho, S. Muraoka, F. Shima, T. Kataoka, K. Kontani, and T. Katada. 2009. Biochemical characterization of missense mutations in the Arf/Arl-family small GTPase Arl6 causing Bardet-Biedl syndrome. Biochem Biophys Res Commun. 381:439-442.

Kosling, S.K., E.K. Fansa, S. Maffini, and A. Wittinghofer. 2018. Mechanism and dynamics of INPP5E transport into and inside the ciliary compartment. Biol Chem. 399:277-292.

Lai, C.K., N. Gupta, X. Wen, L. Rangell, B. Chih, A.S. Peterson, J.F. Bazan, L. Li, and S.J. Scales. 2011. Functional characterization of putative cilia genes by high-content analysis. Mol Biol Cell. 22:1104-1119.

Larkins, C.E., G.D. Aviles, M.P. East, R.A. Kahn, and T. Caspary. 2011. Arl13b regulates ciliogenesis and the dynamic localization of Shh signaling proteins. Mol Biol Cell. 22:4694-4703.

Lechtreck, K.F. 2015. IFT-Cargo Interactions and Protein Transport in Cilia. Trends Biochem Sci. 40:765-778.

Leipe, D.D., Y.I. Wolf, E.V. Koonin, and L. Aravind. 2002. Classification and evolution of P-loop GTPases and related ATPases. J Mol Biol. 317:41-72.

Li, J.B., J.M. Gerdes, C.J. Haycraft, Y. Fan, T.M. Teslovich, H. May-Simera, H. Li, O.E. Blacque, L. Li, C.C. Leitch, R.A. Lewis, J.S. Green, P.S. Parfrey, M.R. Leroux, W.S. Davidson, P.L. Beales, L.M. GuayWoodford, B.K. Yoder, G.D. Stormo, N. Katsanis, and S.K. Dutcher. 2004. Comparative genomics identifies a flagellar and basal body proteome that includes the BBS5 human disease gene. Cell. 117:541-552.

Li, L., S. Wang, H. Wang, S.K. Sahu, B. Marin, H. Li, Y. Xu, H. Liang, Z. Li, S. Cheng, T. Reder, Z. Çebi, S. Wittek, M. Petersen, B. Melkonian, H. Du, H. Yang, J. Wang, G.K. Wong, X. Xu, X. Liu, Y. Van de Peer, 
M. Melkonian, and H. Liu. 2020. The genome of Prasinoderma coloniale unveils the existence of a third phylum within green plants. Nature ecology \& evolution. 4:1220-1231.

Li, X., Y. Li, S. Li, H. Li, C. Yang, and J. Lin. 2021. The role of Shh signalling pathway in central nervous system development and related diseases. Cell Biochem Funct. 39:180-189.

Livak, K.J., and T.D. Schmittgen. 2001. Analysis of relative gene expression data using real-time quantitative PCR and the 2(-Delta Delta C(T)) Method. Methods. 25:402-408.

Luck, K., D.K. Kim, L. Lambourne, K. Spirohn, B.E. Begg, W. Bian, R. Brignall, T. Cafarelli, F.J. Campos-Laborie, B. Charloteaux, D. Choi, A.G. Coté, M. Daley, S. Deimling, A. Desbuleux, A. Dricot, M. Gebbia, M.F. Hardy, N. Kishore, J.J. Knapp, I.A. Kovács, I. Lemmens, M.W. Mee, J.C. Mellor, C. Pollis, C. Pons, A.D. Richardson, S. Schlabach, B. Teeking, A. Yadav, M. Babor, D. Balcha, O. Basha, C. Bowman-Colin, S.F. Chin, S.G. Choi, C. Colabella, G. Coppin, C. D'Amata, D. De Ridder, S. De Rouck, M. Duran-Frigola, H. Ennajdaoui, F. Goebels, L. Goehring, A. Gopal, G. Haddad, E. Hatchi, M. Helmy, Y. Jacob, Y. Kassa, S. Landini, R. Li, N. van Lieshout, A. MacWilliams, D. Markey, J.N. Paulson, S. Rangarajan, J. Rasla, A. Rayhan, T. Rolland, A. San-Miguel, Y. Shen, D. Sheykhkarimli, G.M. Sheynkman, E. Simonovsky, M. Taşan, A. Tejeda, V. Tropepe, J.C. Twizere, Y. Wang, R.J. Weatheritt, J. Weile, Y. Xia, X. Yang, E. Yeger-Lotem, Q. Zhong, P. Aloy, G.D. Bader, J. De Las Rivas, S. Gaudet, T. Hao, J. Rak, J. Tavernier, D.E. Hill, M. Vidal, F.P. Roth, and M.A. Calderwood. 2020. A reference map of the human binary protein interactome. Nature. 580:402-408.

Maddison, W.P. 2000. Testing character correlation using pairwise comparisons on a phylogeny. J Theor Biol. 202:195-204.

Mariani, L.E., M.F. Bijlsma, A.A. Ivanova, S.K. Suciu, R.A. Kahn, and T. Caspary. 2016. Arl13b regulates Shh signaling from both inside and outside the cilium. Mol Biol Cell. 27:3780-3790.

Marley, A., R.W. Choy, and M. von Zastrow. 2013. GPR88 reveals a discrete function of primary cilia as selective insulators of GPCR cross-talk. PLoS One. 8:e70857.

May-Simera, H., K. Nagel-Wolfrum, and U. Wolfrum. 2017. Cilia - The sensory antennae in the eye. Prog Retin Eye Res. 60:144-180.

Merchant, S.S., S.E. Prochnik, O. Vallon, E.H. Harris, S.J. Karpowicz, G.B. Witman, A. Terry, A. Salamov, L.K. Fritz-Laylin, L. Marechal-Drouard, W.F. Marshall, L.H. Qu, D.R. Nelson, A.A. Sanderfoot, M.H. Spalding, V.V. Kapitonov, Q. Ren, P. Ferris, E. Lindquist, H. Shapiro, S.M. Lucas, J. Grimwood, J. Schmutz, P. Cardol, H. Cerutti, G. Chanfreau, C.L. Chen, V. Cognat, M.T. Croft, R. Dent, S. Dutcher, E. Fernandez, H. Fukuzawa, D. Gonzalez-Ballester, D. Gonzalez-Halphen, A. Hallmann, M. Hanikenne, M. Hippler, W. Inwood, K. Jabbari, M. Kalanon, R. Kuras, P.A. Lefebvre, S.D. Lemaire, A.V. Lobanov, M. Lohr, A. Manuell, I. Meier, L. Mets, M. Mittag, T. Mittelmeier, J.V. Moroney, J. Moseley, C. Napoli, A.M. Nedelcu, K. Niyogi, S.V. Novoselov, I.T. Paulsen, G. Pazour, S. Purton, J.P. Ral, D.M. Riano-Pachon, W. Riekhof, L. Rymarquis, M. Schroda, D. Stern, J. Umen, R. Willows, N. Wilson, S.L. Zimmer, J. Allmer, J. Balk, K. Bisova, C.J. Chen, M. Elias, K. Gendler, C. Hauser, M.R. Lamb, H. Ledford, J.C. Long, J. Minagawa, M.D. Page, J. Pan, W. Pootakham, S. Roje, A. Rose, E. Stahlberg, A.M. Terauchi, P. Yang, S. Ball, C. Bowler, C.L. Dieckmann, V.N. Gladyshev, P. Green, R. Jorgensen, S. Mayfield, B. Mueller-Roeber, S. Rajamani, R.T. Sayre, P. Brokstein, et al. 2007. The Chlamydomonas genome reveals the evolution of key animal and plant functions. Science. 318:245-250.

Moran, J., P.G. McKean, and M.L. Ginger. 2014. Eukaryotic Flagella: Variations in Form, Function, and Composition during Evolution. BioScience. 64:1103-1114.

Mourão, A., A.R. Nager, M.V. Nachury, and E. Lorentzen. 2014. Structural basis for membrane targeting of the BBSome by ARL6. Nat Struct Mol Biol. 21:1035-1041.

Mukhopadhyay, S., X. Wen, B. Chih, C.D. Nelson, W.S. Lane, S.J. Scales, and P.K. Jackson. 2010. TULP3 bridges the IFT-A complex and membrane phosphoinositides to promote trafficking of $\mathrm{G}$ protein-coupled receptors into primary cilia. Genes Dev. 24:2180-2193.

Nachury, M.V. 2018. The molecular machines that traffic signaling receptors into and out of cilia. Curr Opin Cell Biol. 51:124-131.

Nachury, M.V., and D.U. Mick. 2019. Establishing and regulating the composition of cilia for signal transduction. Nat Rev Mol Cell Biol. 20:389-405. 
Nevers, Y., M.K. Prasad, L. Poidevin, K. Chennen, A. Allot, A. Kress, R. Ripp, J.D. Thompson, H. Dollfus, O. Poch, and O. Lecompte. 2017. Insights into Ciliary Genes and Evolution from Multi-Level Phylogenetic Profiling. Mol Biol Evol. 34:2016-2034.

Ocbina, P.J., J.T. Eggenschwiler, I. Moskowitz, and K.V. Anderson. 2011. Complex interactions between genes controlling trafficking in primary cilia. Nat Genet. 43:547-553.

Pazour, G.J., S.A. Baker, J.A. Deane, D.G. Cole, B.L. Dickert, J.L. Rosenbaum, G.B. Witman, and J.C. Besharse. 2002. The intraflagellar transport protein, IFT88, is essential for vertebrate photoreceptor assembly and maintenance. The Journal of cell biology. 157:103-113.

Piette, B.L., N. Alerasool, Z.Y. Lin, J. Lacoste, M.H.Y. Lam, W.W. Qian, S. Tran, B. Larsen, E. Campos, J. Peng, A.C. Gingras, and M. Taipale. 2021. Comprehensive interactome profiling of the human Hsp70 network highlights functional differentiation of J domains. Mol Cell. 81:2549-2565 e2548.

Placzek, M., and J. Briscoe. 2018. Sonic hedgehog in vertebrate neural tube development. Int J Dev Biol. 62:225234.

Poole, C.A., C.G. Jensen, J.A. Snyder, C.G. Gray, V.L. Hermanutz, and D.N. Wheatley. 1997. Confocal analysis of primary cilia structure and colocalization with the Golgi apparatus in chondrocytes and aortic smooth muscle cells. Cell Biol Int. 21:483-494.

Qiu, H., S. Fujisawa, S. Nozaki, Y. Katoh, and K. Nakayama. 2021. Interaction of INPP5E with ARL13B is essential for its ciliary membrane retention but dispensable for its ciliary entry. Biol Open. 10.

Quidwai, T., E.A. Hall, M.A. Keighren, W. Leng, P. Kiesel, J.N. Wells, L.C. Murphy, J.A. Marsh, G. Pigino, and P. Mill. 2021. A WDR35-dependent coatomer transports ciliary membrane proteins from the Golgi to the cilia. bioRxiv:2020.2012.2022.423978.

Roepman, R., and U. Wolfrum. 2007. Protein networks and complexes in photoreceptor cilia. Subcell Biochem. 43:209-235.

Rolland, T., M. Tasan, B. Charloteaux, S.J. Pevzner, Q. Zhong, N. Sahni, S. Yi, I. Lemmens, C. Fontanillo, R. Mosca, A. Kamburov, S.D. Ghiassian, X. Yang, L. Ghamsari, D. Balcha, B.E. Begg, P. Braun, M. Brehme, M.P. Broly, A.R. Carvunis, D. Convery-Zupan, R. Corominas, J. Coulombe-Huntington, E. Dann, M. Dreze, A. Dricot, C. Fan, E. Franzosa, F. Gebreab, B.J. Gutierrez, M.F. Hardy, M. Jin, S. Kang, R. Kiros, G.N. Lin, K. Luck, A. MacWilliams, J. Menche, R.R. Murray, A. Palagi, M.M. Poulin, X. Rambout, J. Rasla, P. Reichert, V. Romero, E. Ruyssinck, J.M. Sahalie, A. Scholz, A.A. Shah, A. Sharma, Y. Shen, K. Spirohn, S. Tam, A.O. Tejeda, S.A. Trigg, J.C. Twizere, K. Vega, J. Walsh, M.E. Cusick, Y. Xia, A.L. Barabasi, L.M. lakoucheva, P. Aloy, J. De Las Rivas, J. Tavernier, M.A. Calderwood, D.E. Hill, T. Hao, F.P. Roth, and M. Vidal. 2014. A proteome-scale map of the human interactome network. Cell. 159:12121226.

Satir, P., and S.T. Christensen. 2007. Overview of structure and function of mammalian cilia. Annu Rev Physiol. 69:377-400.

Schiavon, C.R., R.E. Turn, L.E. Newman, and R.A. Kahn. 2019. ELMOD2 regulates mitochondrial fusion in a mitofusin-dependent manner, downstream of ARL2. Mol Biol Cell. 30:1198-1213.

Schrick, J.J., P. Vogel, A. Abuin, B. Hampton, and D.S. Rice. 2006. ADP-ribosylation factor-like 3 is involved in kidney and photoreceptor development. Am J Pathol. 168:1288-1298.

Sedmak, T., and U. Wolfrum. 2010. Intraflagellar transport molecules in ciliary and nonciliary cells of the retina. The Journal of cell biology. 189:171-186.

Smits, A.H., F. Ziebell, G. Joberty, N. Zinn, W.F. Mueller, S. Clauder-Munster, D. Eberhard, M. Falth Savitski, P. Grandi, P. Jakob, A.M. Michon, H. Sun, K. Tessmer, T. Burckstummer, M. Bantscheff, L.M. Steinmetz, G. Drewes, and W. Huber. 2019. Biological plasticity rescues target activity in CRISPR knock outs. Nat Methods. 16:1087-1093.

Stephen, L.A., Y. Elmaghloob, and S. Ismail. 2017. Maintaining protein composition in cilia. Biol Chem. 399:111.

Sztul, E., P.W. Chen, J.E. Casanova, J. Cherfils, J.B. Dacks, D.G. Lambright, F.S. Lee, P.A. Randazzo, L.C. Santy, A. Schurmann, I. Wilhelmi, M.E. Yohe, and R.A. Kahn. 2019. ARF GTPases and their GEFs and GAPs: concepts and challenges. Mol Biol Cell. 30:1249-1271. 
Thomas, S., K.J. Wright, S.L. Corre, A. Micalizzi, M. Romani, A. Abhyankar, J. Saada, I. Perrault, J. Amiel, J. Litzler, E. Filhol, N. Elkhartoufi, M. Kwong, J.-L. Casanova, N. Boddaert, W. Baehr, S. Lyonnet, A. Munnich, L. Burglen, N. Chassaing, F. Encha-Ravazi, M. Vekemans, J.G. Gleeson, E.M. Valente, P.K. Jackson, I.A. Drummond, S. Saunier, and T. Attié-Bitach. 2014. A Homozygous PDE6D Mutation in Joubert Syndrome Impairs Targeting of Farnesylated INPP5E Protein to the Primary Cilium. Human Mutation. 35:137-146.

Tice, A.K., D. Zihala, T. Panek, R.E. Jones, E.D. Salomaki, S. Nenarokov, F. Burki, M. Elias, L. Eme, A.J. Roger, A. Rokas, X.X. Shen, J.F.H. Strassert, M. Kolisko, and M.W. Brown. 2021. PhyloFisher: A phylogenomic package for resolving eukaryotic relationships. PLoS Biol. 19:e3001365.

Trojan, P., N. Krauss, H.W. Choe, A. Giessl, A. Pulvermuller, and U. Wolfrum. 2008. Centrins in retinal photoreceptor cells: regulators in the connecting cilium. Prog Retin Eye Res. 27:237-259.

Turn, R.E., M.P. East, R. Prekeris, and R.A. Kahn. 2020. The ARF GAP ELMOD2 acts with different GTPases to regulate centrosomal microtubule nucleation and cytokinesis. Mol Biol Cell. 31:2070-2091.

Turn, R.E., J. Linnert, E.D. Gigante, U. Wolfrum, T. Caspary, and R.A. Kahn. 2021. Roles for ELMOD2 and Rootletin in Ciliogenesis. Mol Biol Cell. 32:800-822.

Vargova, R., J.G. Wideman, R. Derelle, R.A. Kahn, V. Klimes, J.B. Dacks, and M. Elias. 2021. Unanticipated diversity, undiscovered ancient paralogs, and Asgard origins of the ARF GTPase protein family. Genome Biology and Evolution. in press.

Wang, W., B.M. Jack, H.H. Wang, M.A. Kavanaugh, R.L. Maser, and P.V. Tran. 2021. Intraflagellar Transport Proteins as Regulators of Primary Cilia Length. Frontiers in Cell and Developmental Biology. 9.

Ward, H.H., U. Brown-Glaberman, J. Wang, Y. Morita, S.L. Alper, E.J. Bedrick, V.H. Gattone, 2nd, D. Deretic, and A. Wandinger-Ness. 2011. A conserved signal and GTPase complex are required for the ciliary transport of polycystin-1. Mol Biol Cell. 22:3289-3305.

Wiens, C.J., Y. Tong, M.A. Esmail, E. Oh, J.M. Gerdes, J. Wang, W. Tempel, J.B. Rattner, N. Katsanis, H.W. Park, and M.R. Leroux. 2010. Bardet-Biedl syndrome-associated small GTPase ARL6 (BBS3) functions at or near the ciliary gate and modulates Wnt signaling. J Biol Chem. 285:16218-16230.

Wingfield, J.L., K.F. Lechtreck, and E. Lorentzen. 2018. Trafficking of ciliary membrane proteins by the intraflagellar transport/BBSome machinery. Essays Biochem. 62:753-763.

Wittinghofer, A., and I.R. Vetter. 2011. Structure-function relationships of the G domain, a canonical switch motif. Annu Rev Biochem. 80:943-971.

Wolfrum, U. 1991. Distribution of F-actin in the compound eye of the blowfly, Calliphora erythrocephala (Diptera, Insecta). Cell and Tissue Research. 263:399-403.

Yadav, S.P., N.K. Sharma, C. Liu, L. Dong, T. Li, and A. Swaroop. 2016. Centrosomal protein CP110 controls maturation of the mother centriole during cilia biogenesis. Development. 143:1491-1501.

Yang, H., and K. Huang. 2019. Dissecting the Vesicular Trafficking Function of IFT Subunits. Front Cell Dev Biol. 7:352.

Yang, J., J. Gao, M. Adamian, X.H. Wen, B. Pawlyk, L. Zhang, M.J. Sanderson, J. Zuo, C.L. Makino, and T. Li. 2005. The ciliary rootlet maintains long-term stability of sensory cilia. Mol Cell Biol. 25:4129-4137.

Yang, J., and T. Li. 2006. Focus on molecules: rootletin. Experimental eye research. 83:1-2.

Yang, J., X. Liu, G. Yue, M. Adamian, O. Bulgakov, and T. Li. 2002. Rootletin, a novel coiled-coil protein, is a structural component of the ciliary rootlet. The Journal of cell biology. 159:431-440.

Yang, Y.-K., H. Qu, D. Gao, W. Di, H.-W. Chen, X. Guo, Z.-H. Zhai, and D.-Y. Chen. 2011. ARF-like Protein 16 (ARL16) Inhibits RIG-I by Binding with Its C-terminal Domain in a GTP-dependent Manner. Journal of Biological Chemistry. 286:10568-10580.

Zhang, T., S. Li, Y. Zhang, C. Zhong, Z. Lai, and J. Ding. 2009. Crystal structure of the ARL2-GTP-BART complex reveals a novel recognition and binding mode of small GTPase with effector. Structure. 17:602-610.

Zhou, C., L. Cunningham, A.I. Marcus, Y. Li, and R.A. Kahn. 2006. Arl2 and Arl3 regulate different microtubuledependent processes. Mol Biol Cell. 17:2476-2487.

\section{FIGURE LEGENDS}


Figure 1. Phylogenetic distribution of ARL16 and the three previously known cilium-associated ARF family members (ARL3, ARL6, and ARL13) and cilia in eukaryotes. The distribution of the four ARF-like genes and cilium (columns) is depicted by filled blue circle (gene/cilium is present) or empty red circle (gene/cilium is absent or not detected). In three species the status of the cilium is ambiguous (see Materials and methods and Table S1). The sequence data type analyzed to establish the presence/absence of the ARLs in the different taxa is indicated ("genome or transcriptome" means that these two types of resources had to be combined to obtain a full set of the orthologs sought). The absence of any of the four ARLs in taxa represented only by transcriptome assemblies must be considered tentative, as the respective genes may be present but not expressed in the stage used for generating the transcriptome data. The schematic phylogeny on the right reflects the current state of knowledge based on multiple phylogenomic analyses. Monophyletic groups of two or more species uniform as to the presence/absence of the cilium and the four focal ARLs are shown as single branches, with the name of the respective broader taxon and the number of taxa included indicated in square brackets (see Table S1 for the full species list). The relationship at the level of the deepest branches, still not completely settled, is adopted from the most recent and comprehensive phylogenomic analysis (Tice et al., 2021), whereas the root of the tree is indicated following the rooting hypothesis by Derelle, et al. (Derelle et al., 2015). Independent losses of the ARL16 gene as inferred by parsimony reasoning from its distribution of extant taxa are mapped onto the phylogenetic tree.

Figure 2. Characterization of endogenous ARL16 localization in RPE1 cells and human retina. (A) ARL16 localizes to cilia in RPE1 cells, as observed by immunocytochemistry after PFA fixation (see Materials and Methods). RPE1 cells were serum starved for $48 \mathrm{hrs}$ and stained for ARL16 (green), Ac-Tu (red), and Hoechst (blue). (B) Indirect co-immunolabeling of ARL16 (red) and centrin 3 (green), a common marker for the connecting cilium (CC) and the basal body (BB), of a human retina revealed immunofluorescence of ARL16 in the ciliary region (CR) and the inner segment (IS) of retinal photoreceptor cells. Photoreceptor outer segments (OS), DAPIstained outer and inner nuclear layer (blue, ONL, INL), and outer and inner plexiform layer (OPL, IPL) did not show substantial staining. Asterisk $(*)$ indicates anti-ARL16 blood vessel staining. No staining was observed in controls lacking ARL16 antibodies. Scheme of ARL16 localization in photoreceptor cells. ARL16 is localized to periciliary/basal body region (BB) and in the IS of human photoreceptor cells. Scale bars: $A, 10 \mu \mathrm{m}$. B, $15 \mu \mathrm{m}$ and $5 \mu \mathrm{m}$ (higher magnification).

Figure 3: Deletion of Arl16 causes defects in ciliogenesis. (A) WT and Arl16 KO MEFs were serum starved with percentage of ciliated cells manually counted. For each cell line ( $n=2$ WT and $n=5$ KO), 100 cells were scored at each time point in triplicate and averaged. Multiple unpaired t-tests, false discovery rate $Q=1 \%$, Twostage step-up (Benjamini, Krieger, and Yekutieli). 24h $\mathrm{p}=0.017503,48 \mathrm{~h} \mathrm{p}=0.020865,72 \mathrm{~h} \mathrm{p}=0.076598$ (B) Arl16 $\mathrm{KO}$ cells fail to ciliate as efficiently as WT cells, particularly at earlier timepoints after serum starvation. WT and Arl16 KO MEFs serum starved for $24 \mathrm{~h}$ or $72 \mathrm{~h}$ and stained for Ac-Tu. Scale bar $=10 \mu \mathrm{m}, 60 \mathrm{x}$. (C) Expression of ARL16-myc restores ciliation in Arl16 KO cells after $24 \mathrm{~h}$ of serum starvation. Values for untransfected cells are equivalent to the data from panel $\mathrm{A}$ at $24 \mathrm{~h}$ of serum starvation. Ciliation of transfected cells was scored as in panel $A(\mathrm{~N}=2 \times 50$ transfected cells from one WT line and $2 \mathrm{KO}$ lines). Two way ANOVA Tukey's multiple comparisons (Untransfected WT vs KO $p=0.0315$, WT transfected vs untransfected $p=0.1349$, KO Untransfected vs Transfected $p=0.0277$, Transfected WT vs KO $p=0.3133$ ) (D) Arl16 KO cilia are longer than WT cilia. Cells were serum starved for $48 \mathrm{~h}$ before fixing and staining for Ac-Tu, and imaged at 100X. Scale bar $5 \mu \mathrm{m}$. (E) Cilium lengths were measured in WT and Arl16 KO cells using the CiliaQ Plugin in FIJI. N=26 cilia for 1 WT and 2 KO lines. T-test $\mathrm{P}=0.0023$.

Figure 4: Arl16 KO cells have reduced recruitment of several ciliary proteins. (A) $A R L 13 B$ levels are reduced in Arl16 KO cilia. Cells were serum starved for 48h and stained for Ac-Tu and ARL13B. (B) Quantification of ARL13B positive cilia in WT and Arl16 KO lines. N=3x100 cilia X $5 \mathrm{KO}$ lines or 2 WT lines. Cilia were identified using the Ac-Tu channel. Presence of ARL13B was scored by eye. Normal ARL13B staining was defined as staining that was readily apparent independent of the Ac-Tu channel. Reduced staining was only 
apparent after identification of the cilium using the Ac-Tu channel. T-test. $P=0.0002$. (C) ARL3 is reduced in Arl16 KO cilia. Cells were serum starved for $48 \mathrm{~h}$ and stained for Ac-Tu and ARL3. (D) Quantification of C. $\mathrm{N}=3 \times 100$ cilia $X 5 \mathrm{KO}$ lines or 2 WT lines. Cilia were identified using the Ac-Tu channel. Presence of ARL3 was scored by eye. T-test $p=0.0002$ (E) INPP5E is absent from Arl16 KO cilia. Cells were serum starved for $48 \mathrm{~h}$ and stained for Ac-Tu and INPP5E. (F) Quantification of E. N=3x100 cilia X 5 KO lines or 2 WT lines. Cilia were identified using the Ac-Tu channel. Presence of INPP5E was scored by eye. T-test $p<0.0001$. (G) AC3 is reduced in Arl16 KO cilia. Cells were serum starved for $48 \mathrm{~h}$ and stained for Ac-Tu and AC3. (H) Quantification of G. $\mathrm{N}=3 \times 100$ cilia $X 5 \mathrm{KO}$ lines or 2 WT lines. Cilia were identified using the Ac-Tu channel. Presence of AC3 was scored by eye. T-test $p=0.0098$. Scale bar $=5 \mu \mathrm{m} 100 x$ for all images.

Figure 5: Arl16 KO cells have defects in Hh signaling. (A) SMO is lost from cilia in Arl16 KO lines. Cells were serum starved for $48 \mathrm{~h}$ and stained for Ac-Tu and SMO. (B) Quantification of A. N=2x100 cilia X 2 KO lines and 1 WT line per condition. (C) Arl16 KO MEFs show reduced Shh-stimulated Gli1 and Ptch1 transcriptional response compared to WT cells. Cells were collected $24 \mathrm{~h}$ after Shh treatment, and levels of Gli1 and Ptch1 mRNA were determined using qPCR. Bar graphs indicate normalized mRNA expression with data presented as mean fold change \pm SEM. $\mathrm{N}=2$ WT lines and $4 \mathrm{KO}$ lines.

Figure 6: IFT140 is lost from cilia in Arl16 KO cells, but other IFTs are unchanged. In each case, cells were serum starved for $48 \mathrm{~h}$ and then fixed and (top) stained with Ac-Tu and either IFT140 (A), IFT144 (B), IFT88 (C), or IFT81 (D) and imaged at 100x magnification. Quantification was performed in each case in duplicated for 100 cilia in WT ( $\mathrm{N}=2$ cell lines) and Arl16 KO lines ( $\mathrm{N}=2$ lines). (A) IFT140 is lost from cilia in Arl16 KO lines. (B) IFT144 staining in cilia is unchanged between WT and Arl16 KO lines. $\mathrm{p}=0.1056$. (C) IFT88 is not altered in Arl16 $\mathrm{KO}$ cilia from that in WT cells. $\mathrm{p}=0.6985$. (D) IFT81 is present in Arl16 KO cilia as it is in WT cilia. $\mathrm{p}=0.6855$. Scale bar $=5 \mu \mathrm{m}$.

Figure 7: IFT140 and INPP5E accumulate at the Golgi of Arl16 KO lines. (A) Cells were serum starved for 24h and stained for IFT140 and GM130. IFT140 staining is diffuse in WT cells, but clearly enriched at the Golgi in Arl16 KOs. Asterisks indicate transfected cell. Scale bar $10 \mu \mathrm{m}$. (B) Expression of ARL16-myc reverses IFT140 accumulation in the Golgi. Cells were transfected with ARL16-myc, serum starved for $24 \mathrm{~h}$, and stained for myc, GM130, and IFT140. (C) IFT20 localizes to Golgi indistinguishably in WT and Arl16 KO cells. Cells were serum starved for $24 \mathrm{~h}$ and stained for IFT20. (D) Serum starved cells were fixed and stained after $24 \mathrm{~h}$ and stained for INPP5E and GM130. INPP5E co-staining with the Golgi marker GM130 is very strong while there was no evidence of changes in overall Golgi morphology.

Figure 8: Cells deleted for Pde6d display no defects in ciliation but loss of INPP5E from cilia with its accumulation at Golgi. (A) Cells were serum starved for $24 \mathrm{~h}$, stained for Ac-Tu, and scored for ciliation as described in Materials and Methods. $\mathrm{N}=2 \times 100$ cells for $1 \mathrm{WT}$ and $2 \mathrm{KO}$ lines. T-test $\mathrm{p}=0.5918$. (B) ARL13B and (C) ARL3 localize normally to Pde6d KO cilia. (D) INPP5E is lost from Pde6d KO cilia. (E) IFT140 localizes normally to cilia of Pde6dKO cells. (F) INPP5E is absent from cilia of Pde6d KO lines. Cells were serum starved for 24h and stained for Ac-Tu and INPP5E. (G) INPP5E accumulates in the Golgi of Pde6d KO lines. Cells were serum starved for $24 \mathrm{~h}$ and stained for INPP5E and GM130. All images collected using 100x objective and scale bars $=5 \mu \mathrm{m}$.

Figure 9: Model for ARL16 in ciliogenesis and Golgi to cilium traffic. Ciliogenesis: In WT cells induced to ciliate, CEP164 is recruited to mother centrioles as an early step in the ciliogenesis pathway, with later removal of the CP110 cap and extension of the axoneme. We propose that ARL16 acts after CP110 release to support ciliation such that in its absence ciliation is decreased and/or slowed. Traffic: In WT cells, INPP5E and IFT140 each traffic through the Golgi during transit to cilia. ARL16 supports the export of INPP5E from the Golgi, likely working with PDE6D, to traffic INPP5E from the Golgi to the cilium where it is released by ARL3 and retained by ARL13B. In contrast, IFT140 also requires ARL16, but not INPP5E, to be efficiently exported from the Golgi for 
transit to cilia. In addition, ARL3 and ARL13B fail to be recruited to or retained in cilia. Created with BioRender.com.

Figure S1: ARL16 protein sequences diverge from ARF1 in key motif residues. (A) Alignment of mouse and human ARL16 and human ARF1 protein sequences showing both the long and short human forms and the human ortholog. G motifs are highlighted in red. The mouse and short human forms contain a Cysteine residue at position 2, which may be palmitoylated. Asterisks $\left(^{*}\right)$ indicate residues that differ between human (short) and mouse ARL16 proteins. Alignment was performed using Multalin (http://multalin.toulouse.inra.fr/multalin/multalin.html). (B) Multiple alignment of ARL16 protein sequences from across the eukaryote phylogeny. All identified ARL16 orthologs from the sample of 139 phylogenetically diverse eukaryotes analyzed in this study and (for comparison) the human ARF1 sequence (HsaArf1) were aligned using MAFFT version 7 (https://mafft.cbrc.jp/alignment/server/) and residue conservation pattern was visualized by coloring the alignment using CHROMA Version 1.0 (http://www.lg.ndirect.co.uk/chroma). Four characteristic Gmotifs are framed by red boxes. Note that the ARL16 protein from Physarum polycephalum (PpoArl16) has a very long $\mathrm{C}$-terminal extension consisting of low-complexity repeats; this part of the protein was omitted from the figure. Sequence labels include three-letter species abbreviations indicated in Table S1. Sources of the sequences are provided in Table S2. All the sequences included were checked for accuracy of their inference from nucleotide sequence data, so the apparently unusual features of some of them (insertions, deletions or poor conservation of some regions) most likely reflect the actual primary structure of the respective proteins. In a few cases there is uncertainty concerning the N-terminus of the proteins, as it is possible some (e.g., TruArl16) are artificially extended upstream of the real initiating ( $\mathrm{N}$-terminal) methionine residue. The human sequence (HsaArl16) is represented by the shorter variant.

Figure S2: ARL16 localizes to cytosol and mitochondria. (A) Endogenous ARL16 can be detected at mitochondria in RPE1 cells after methanol fixation, as indicated by colocalization with HSP60. Cells were serum starved for $24 \mathrm{hrs}$ prior to fixation for $5 \mathrm{mins}$ in cold methanol, as described under Materials and Methods. Cells were stained for HSP60 (red) and ARL16 (green). Scale bar $=10 \mu \mathrm{m}$ (60x). (B) Exogenously expressed mouse ARL16-myc and human ARL16 (short) localize primarily to cytosol (diffuse staining) in WT MEFs. Cells were transfected, serum starved for $24 \mathrm{hr}$, fixed with PFA as described in Materials and Methods, then stained for myc or ARL16. Exogenously expressed human ARL16(long) localizes to mitochondria when expressed in MEFs, as indicated by co-localization with HSP60 (bottom panels). Scale bar $=10 \mu \mathrm{m}$. (100x).

Figure S3. Knock out of Arl16 in MEFs and screening of markers. (A) Schematic of mouse Arl16 transcript, with the open reading frame shown below it. Arrows indicate sites targeted by the guide RNAs used for genome editing. Summary of alleles of the 5 Arl $16 \mathrm{KO}$ lines. We obtained three clones from guide 2 and two clones from guide 3. Each of the clones have frameshifting mutations that lead to premature termination of the protein sequence. The black sequence indicates the WT amino acid sequence. Red sequence indicates the predicted amino acids following the frameshift mutations. (B) Mitochondria, actin, microtubules, and nuclei are unchanged in Arl16 KOs. (i) WT and Arl16 KO cells (KO3) were fixed with PFA and stained for HSP60 to mark mitochondria, (ii) with phalloidin (green) to mark the actin cytoskeleton and Hoechst (blue) to mark nuclei, (iii) for tubulin to mark the microtubule cytoskeleton, and (iv-vi) $\beta$-COP, FAPP2, and GBF1 to mark the Golgi. Scale bar $=10 \mu \mathrm{m}$ $(60 x)$.

Figure S4. A number of ciliary features are unchanged between WT and Arl16 KO lines. Arl16 KO cells displayed levels of (A) CEP164 as well as (B) CP110 staining at centrioles comparable to WT MEFs. Cells were serum starved for $24 \mathrm{hr}$ and stained for $\gamma$-tubulin and CEP164 or CP110. The percentage of cells with CEP164 or CP110 at centrosomes was counted. $\mathrm{N}=2 \times 100$ cells $\times 1$ line for WT or 2 lines for Arl16 KOs. (C) Rootlets are fragmented in Arl16 KO cells. WT and Arl16 KO cells were serum starved for $24 \mathrm{hr}$ and stained for rootletin. Scale bar $=10 \mu \mathrm{m}(100 \mathrm{x})$. (D) Quantification of C. $\mathrm{N}=2 \times 100$ cells for 1 WT line and 4 Arl16 KO lines. (E) There is no difference in centrosome separation between WT and Arl16 KO cells. Cells were serum starved for $24 \mathrm{hr}$ and stained for $\gamma$-tubulin. The distance between $\gamma$-tubulin positive puncta (centrosomes) was measured using 
FIJI. (F) SSTR3-GFP reaches cilia indistinguishably in Arl16 KO and WT cells. Cells were transfected with plasmid directing expression of SSTR3-GFP, the next day replated and allowed to recover for $24 \mathrm{~h}$ followed by $24 \mathrm{~h}$ of serum starvation prior to fixation and staining for Ac-Tu. (G) CEP290 localizes normally to the transition zone of Arl16 KO cells. Cells were serum starved for $24 \mathrm{~h}$ and stained for Ac-Tu and CEP290. (H) IFT140 localizes to rootlets and rootlet fragments in WT and Arl16 KO cells. Cells were serum starved for 24h, fixed with methanol, and stained for rootletin and IFT140, Scale bar $=10 \mu \mathrm{m}(100 \mathrm{x})$.

Figure S5: Pde6d CRISPR design. (A) Schematic of mouse Pde6d transcript, with the open reading frame shown below it. Arrows indicate sites targeted by the guide RNAs used for genome editing. (B) Summary of alleles of the 2 Pde6d lines used in these studies. Each of the clones have frameshifting mutations that lead to premature termination of the protein sequence. The black sequence indicates the WT amino acid sequence. Red sequence indicates the predicted amino acids following the frameshift mutation. 


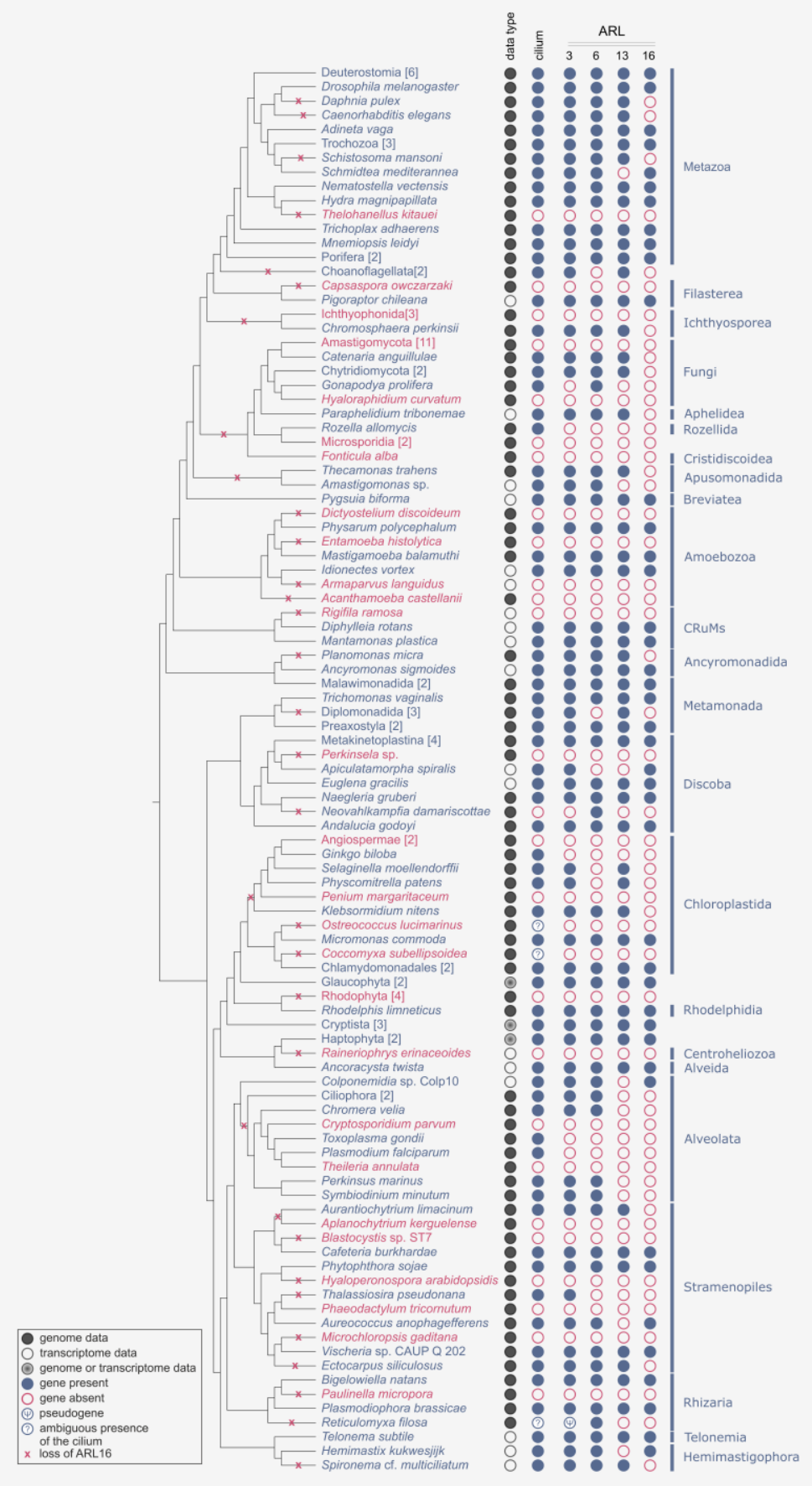

Figure 1 


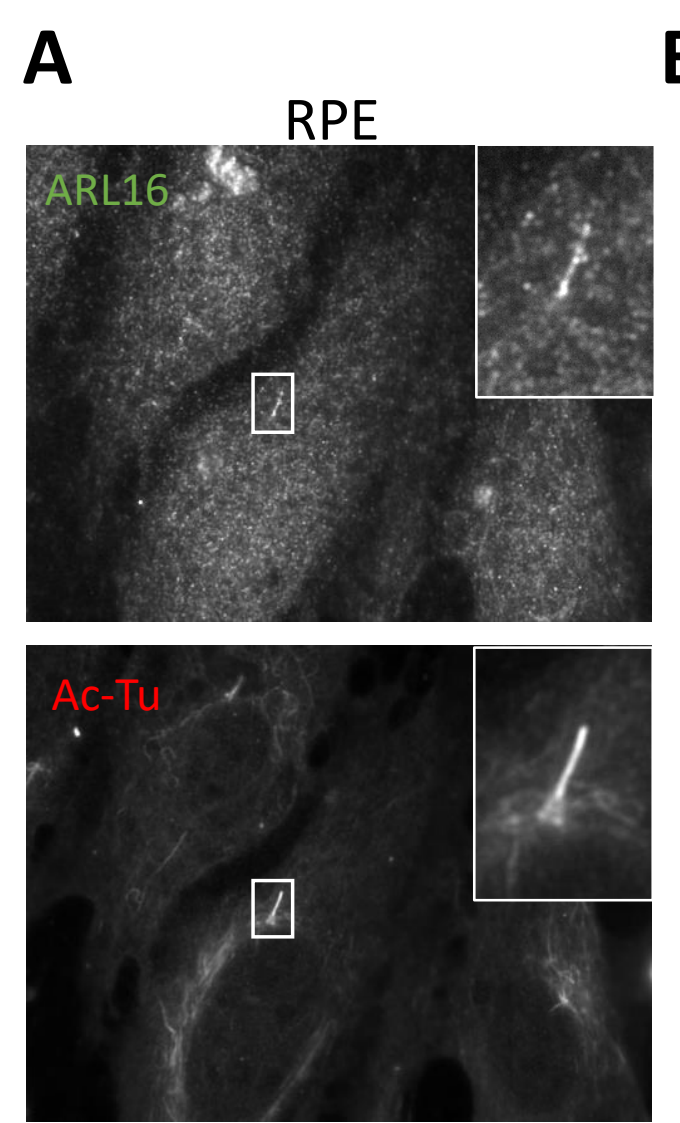

${ }_{\text {DIC/DAPI Arl16 Cen3 merged merged }}$
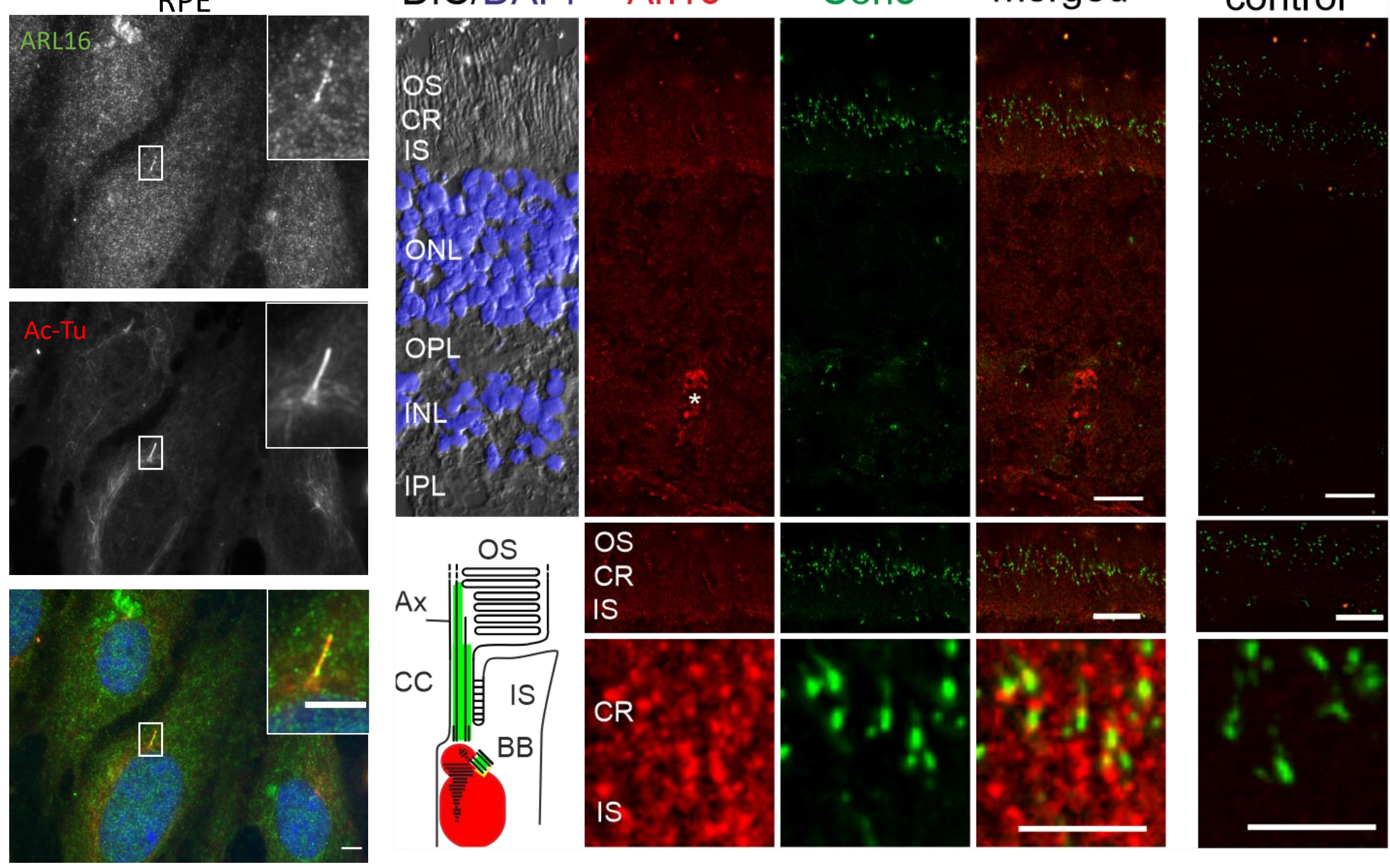

Figure 2 
A

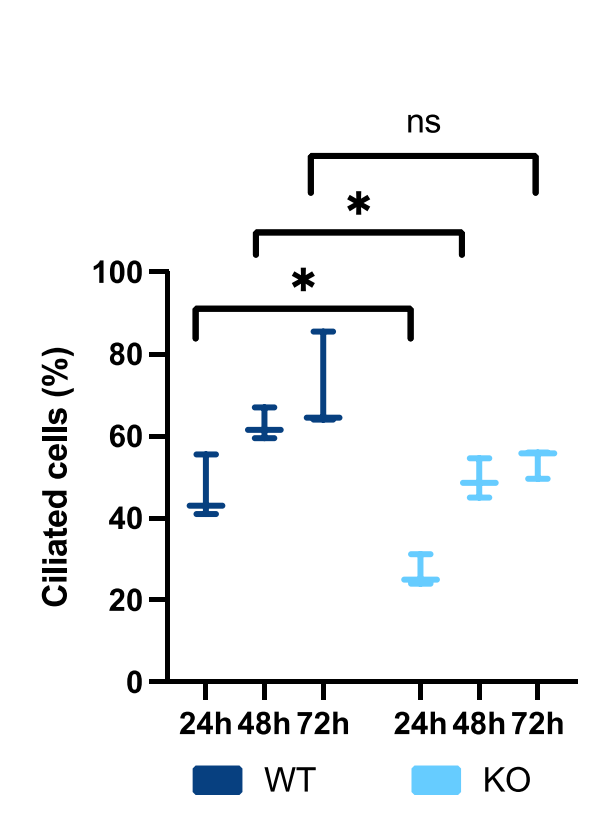

B

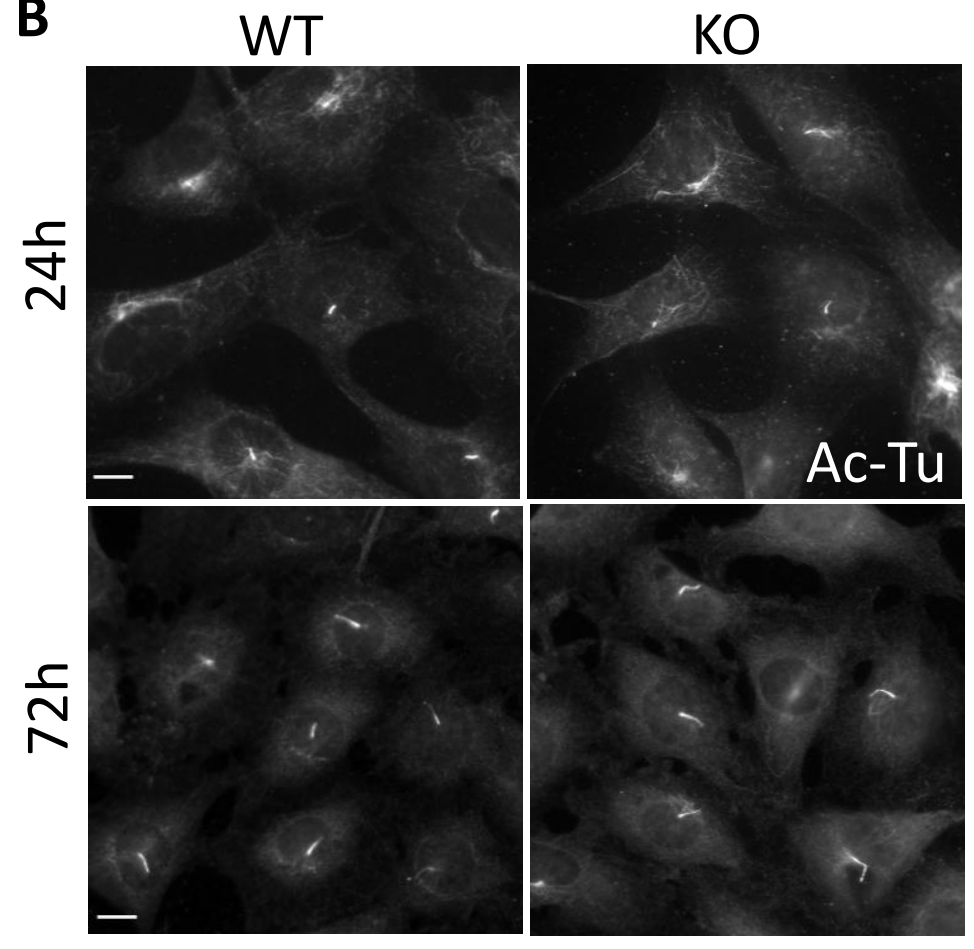

C

D

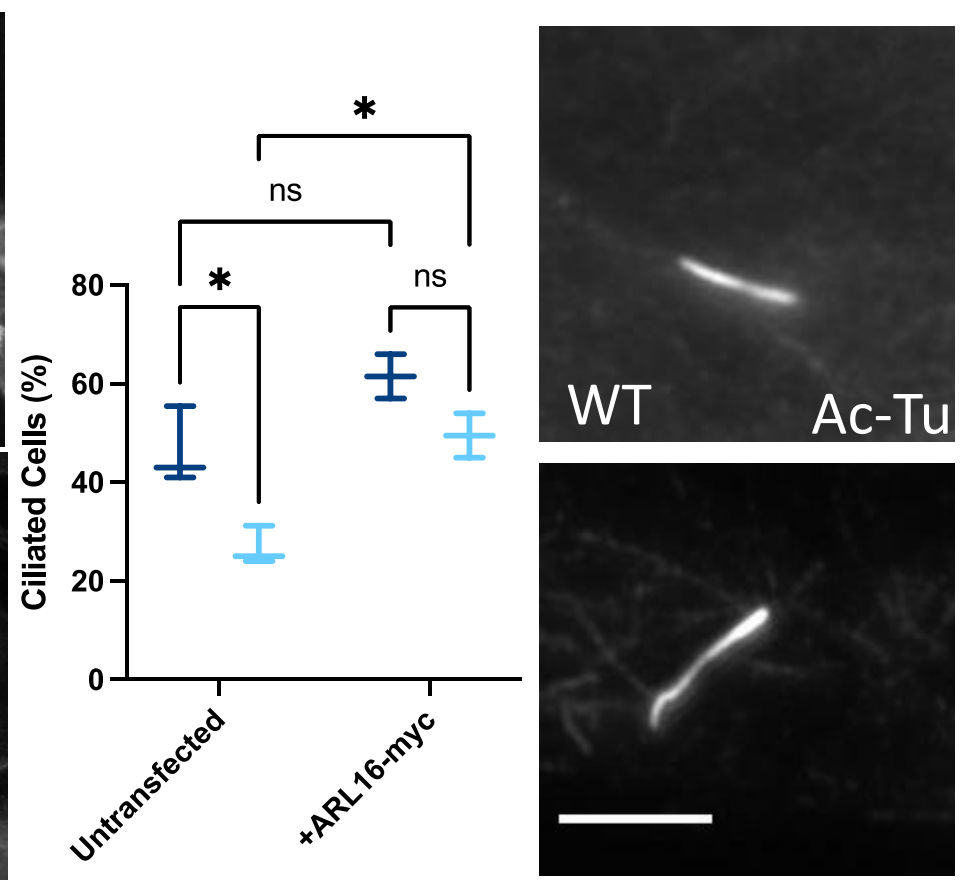

E

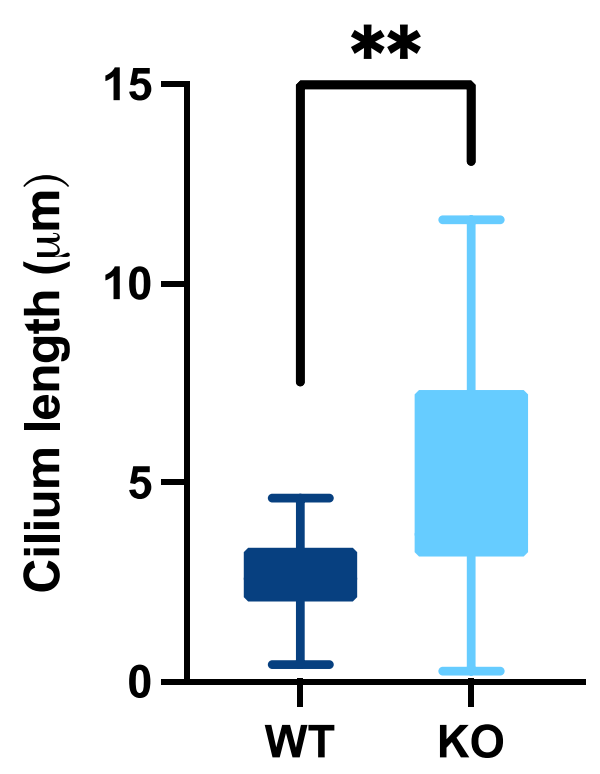

Figure 3 


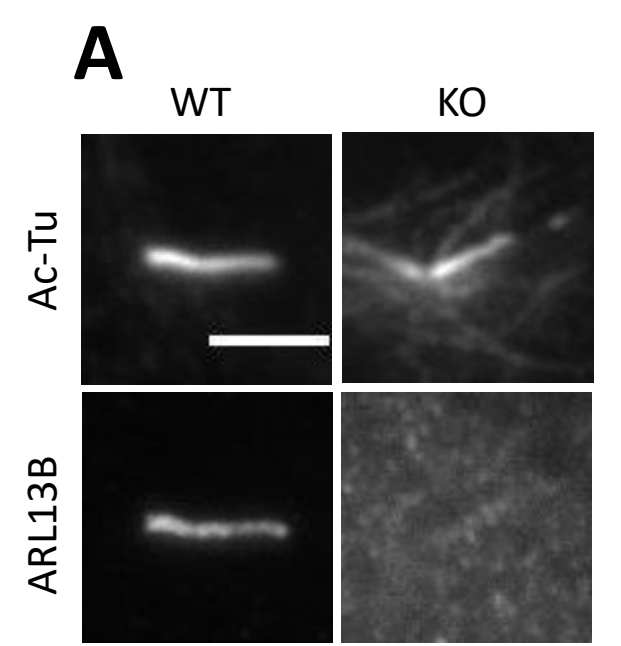

B

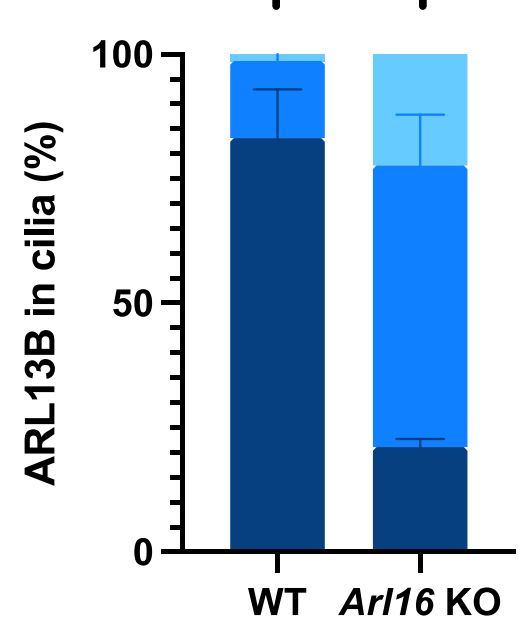

normal $\square$ reduced

absent

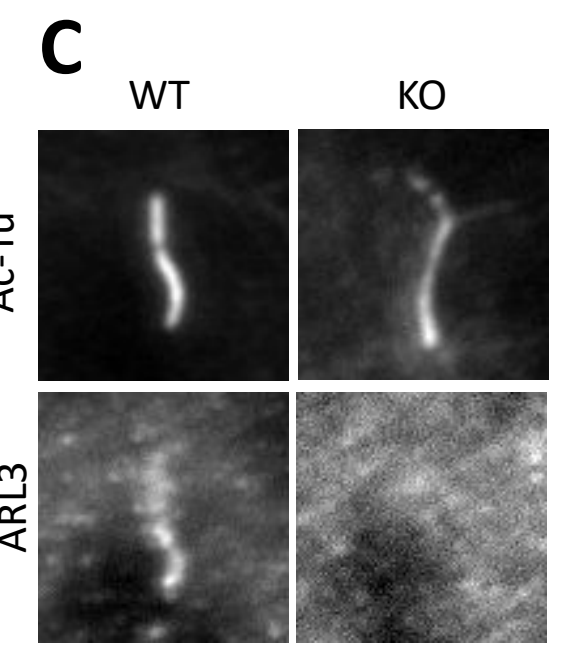

D

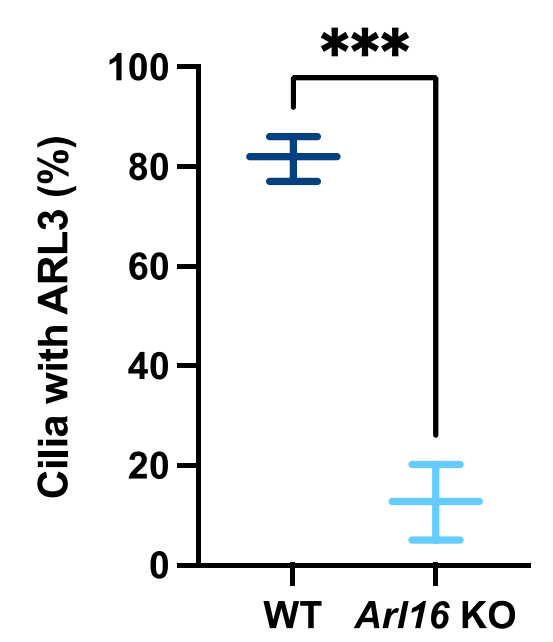

E

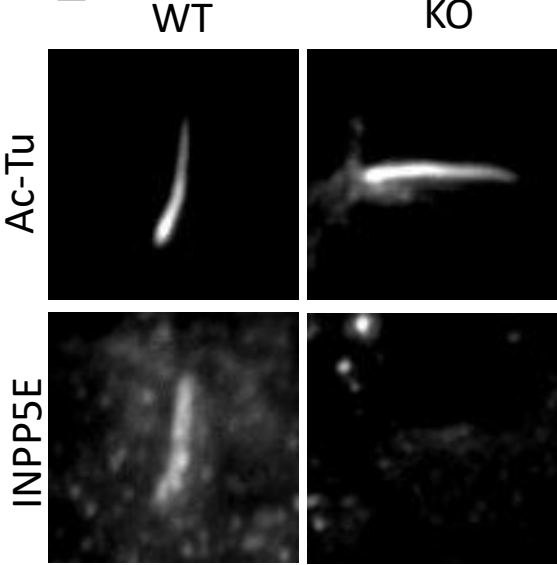

F

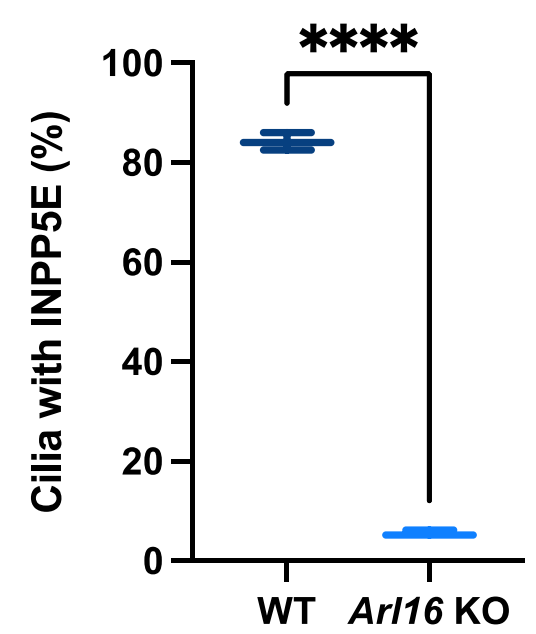

G WT

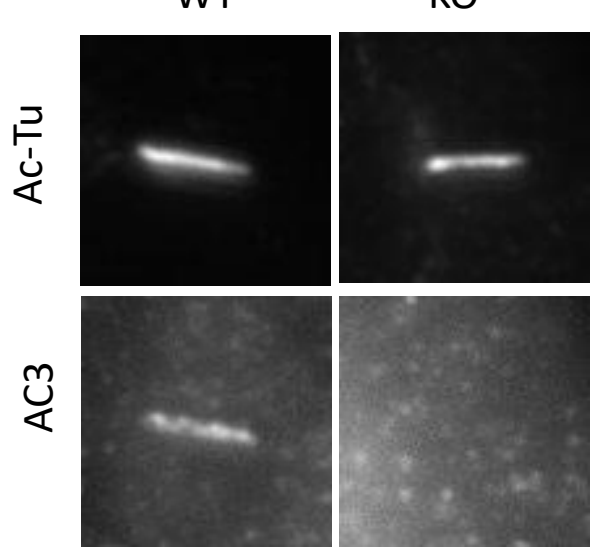

H

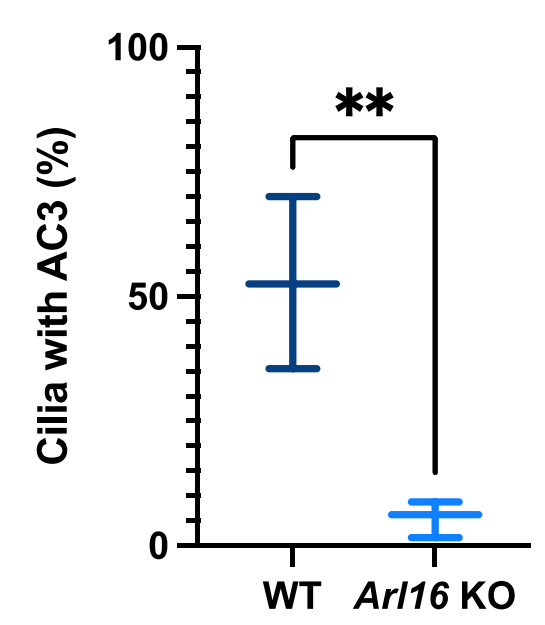

Figure 4 

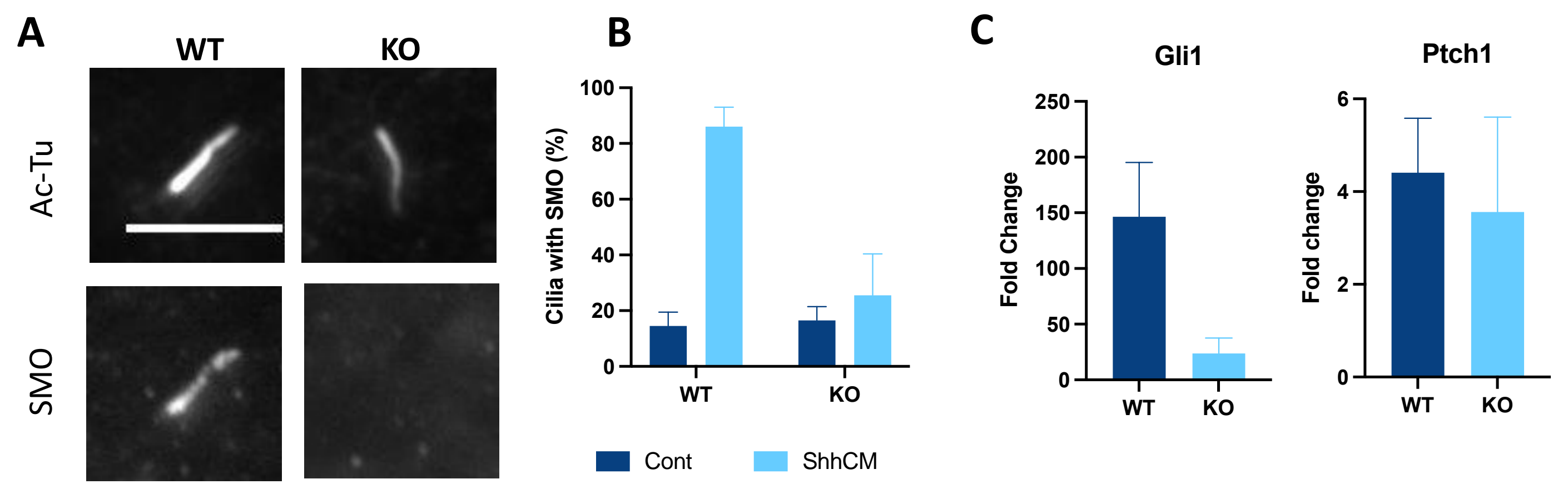

Figure 5 

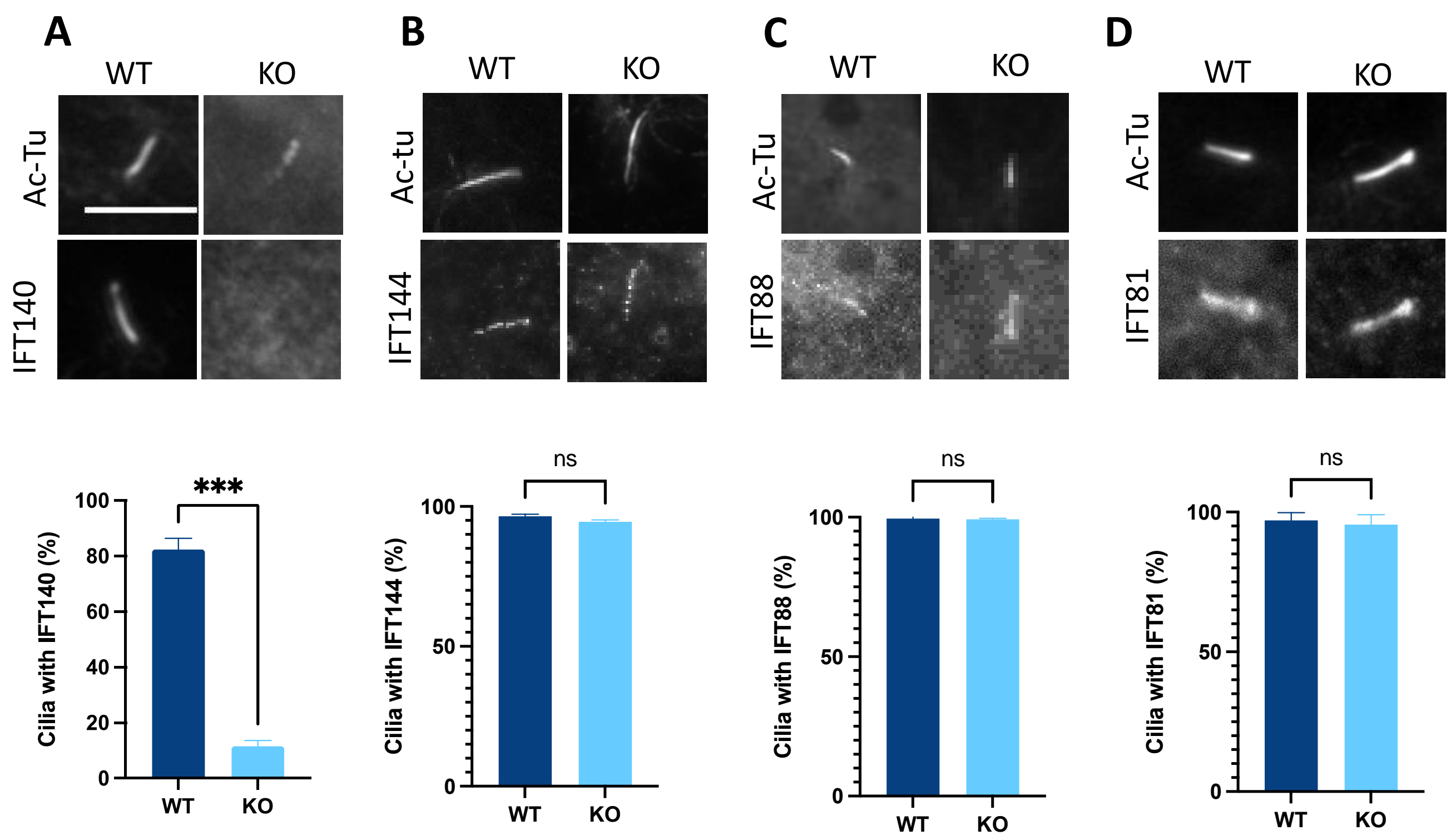

Figure 6 


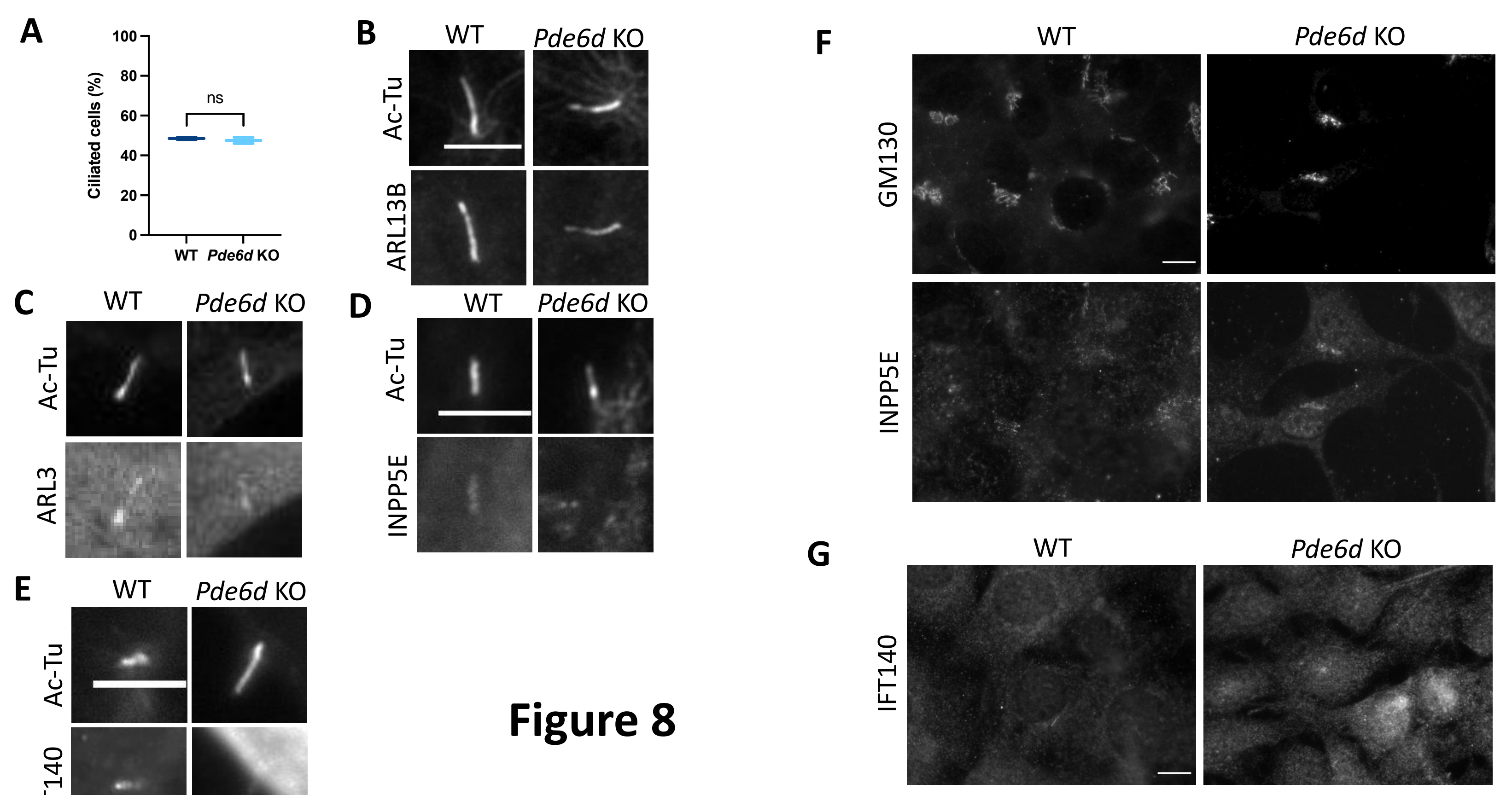




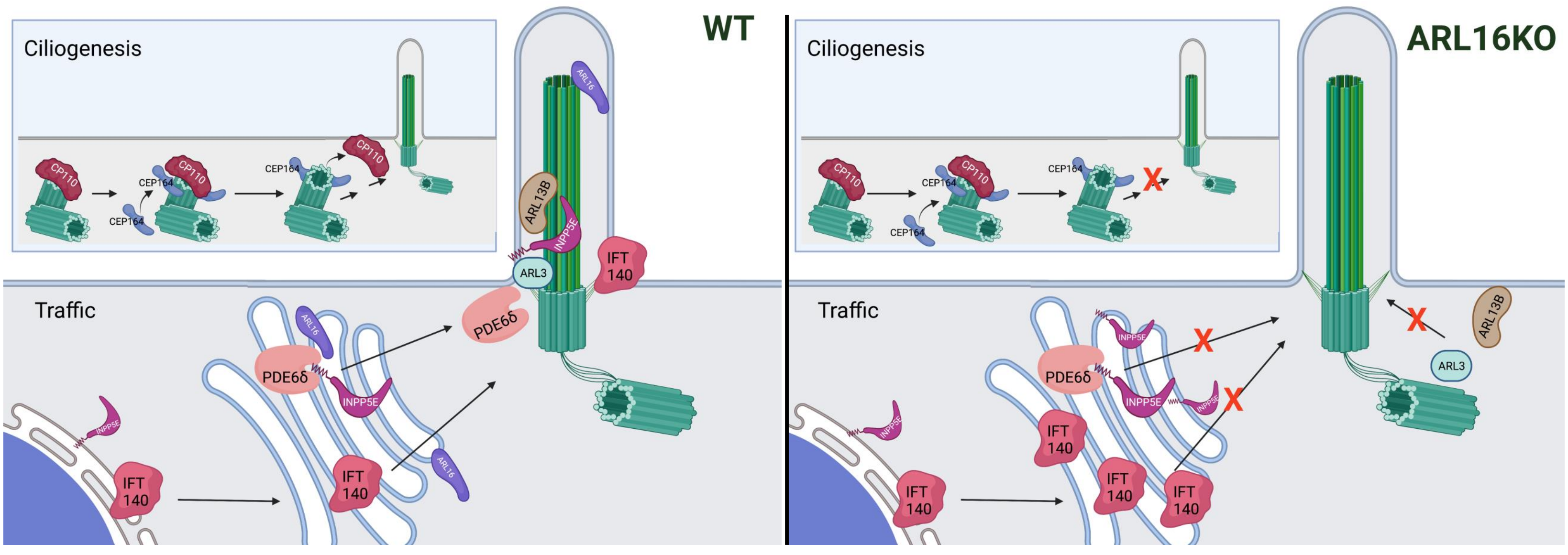

Figure 9 
HSARL16L MRVAGGRALSRGAELRVPGGAKHGMCLLLGATGVGKTLLVKRLQEVSSRDGKGDLGEPPPTRPTVGTNLTDIVAQR.KITIRELGGC..MGPIWSSYYGN - 97 HSARL16S $\ldots \ldots \ldots \ldots \ldots \ldots \ldots \ldots$ MCLLLGATGVGKTLLVKRLQEVSSRDGKGDLGEPPPTRPTVGTNLTDIVAQR.KITIRELGGC..MGPIWSSYYGN - 73 MmARL1 $6 \ldots \ldots \ldots \ldots \ldots \ldots \ldots$ MCLLLGAAGVGKTLLVKRLQKLSSGDGKGDLGEPPPTRPTVGTNLTDIVAHR.KITIRELGGC ..MSPIWSSYYGN -73 HSARF1 ......MGNIFANLFKGLFGKKEMRI LMVGLDAAGKTTILYKLK..........GEIVTTI PTI GENVETVEYKNISFTVWDVGGQDKIRPLWRHYFQN - 84

HSARL16L CRSLLFVMDASDPTQLSASCVQLLGLLSAEQLAEASVLILFNKIDLPCYMSTEEMKSLIRLPDIIACAKQNITTAEISAREGTGLAGVLAWLQATHRAND - 197 HSARL16S CRSLLFVMDASDPTQLSASCVQLLGLLSAEQLAEASVLILFNKIDLPCYMSTEEMKSLIRLPDIIACAKQNITTAEISAREGTGLAGVLAWLQATHRAND - 173 MmARL16 CHSLIFMMDASNPTQLSASCMQLLGLLSAEELEKASVI ILFNKTDIPCYMTMEEMKSLMRLPDIIACAKONITAVE TSARNGTGLATVLLWLQNOHRHSS - 173

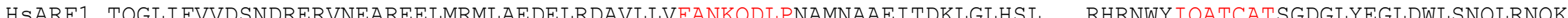$$
\text { * }
$$

$$
\star \star
$$

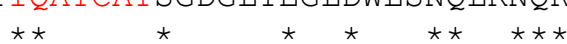

Figure S1 


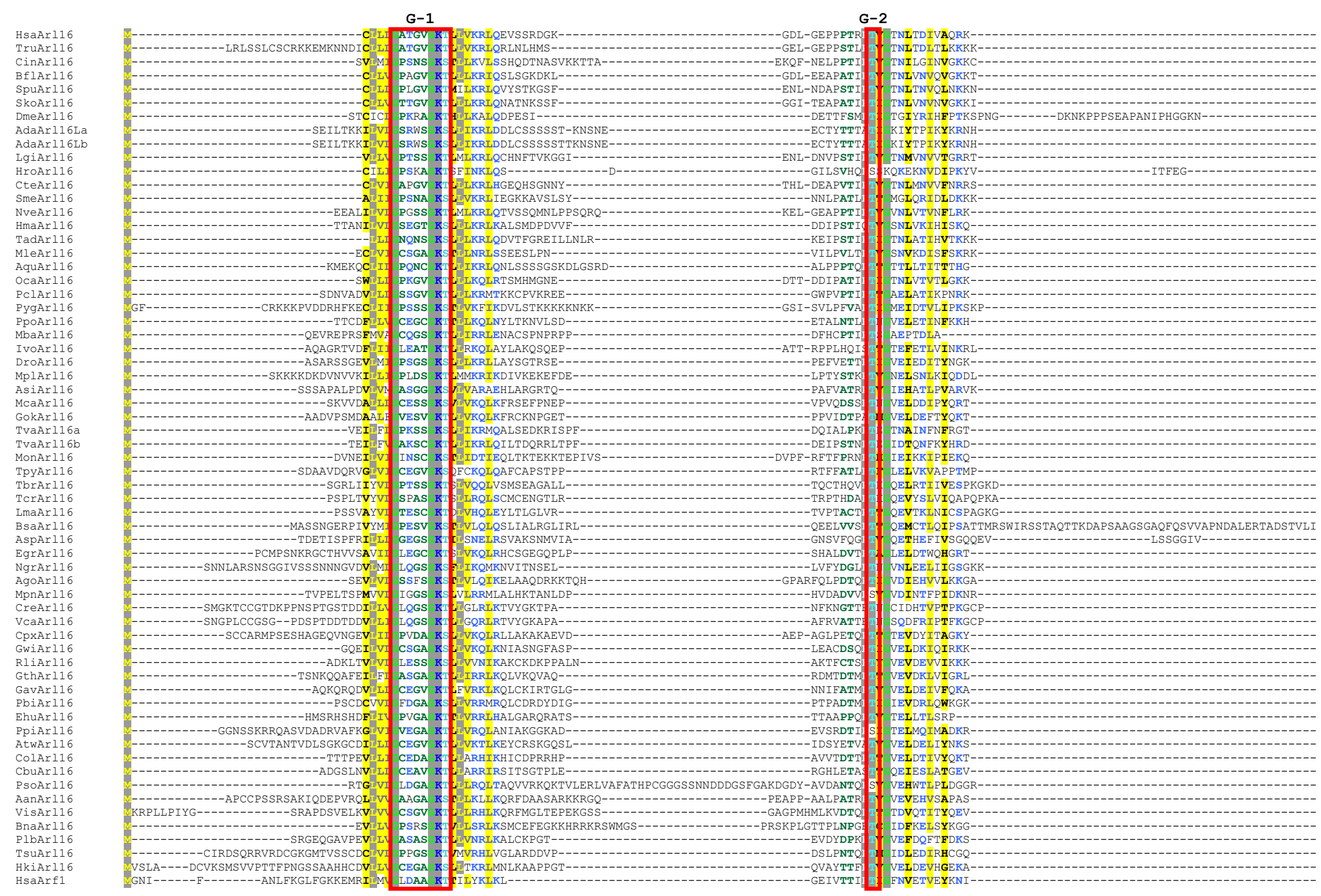

Figure S1 B. Multiple alignment of ARL16 sequences from across the eukaryote phylogeny. The full legend provided at the end of the file. 


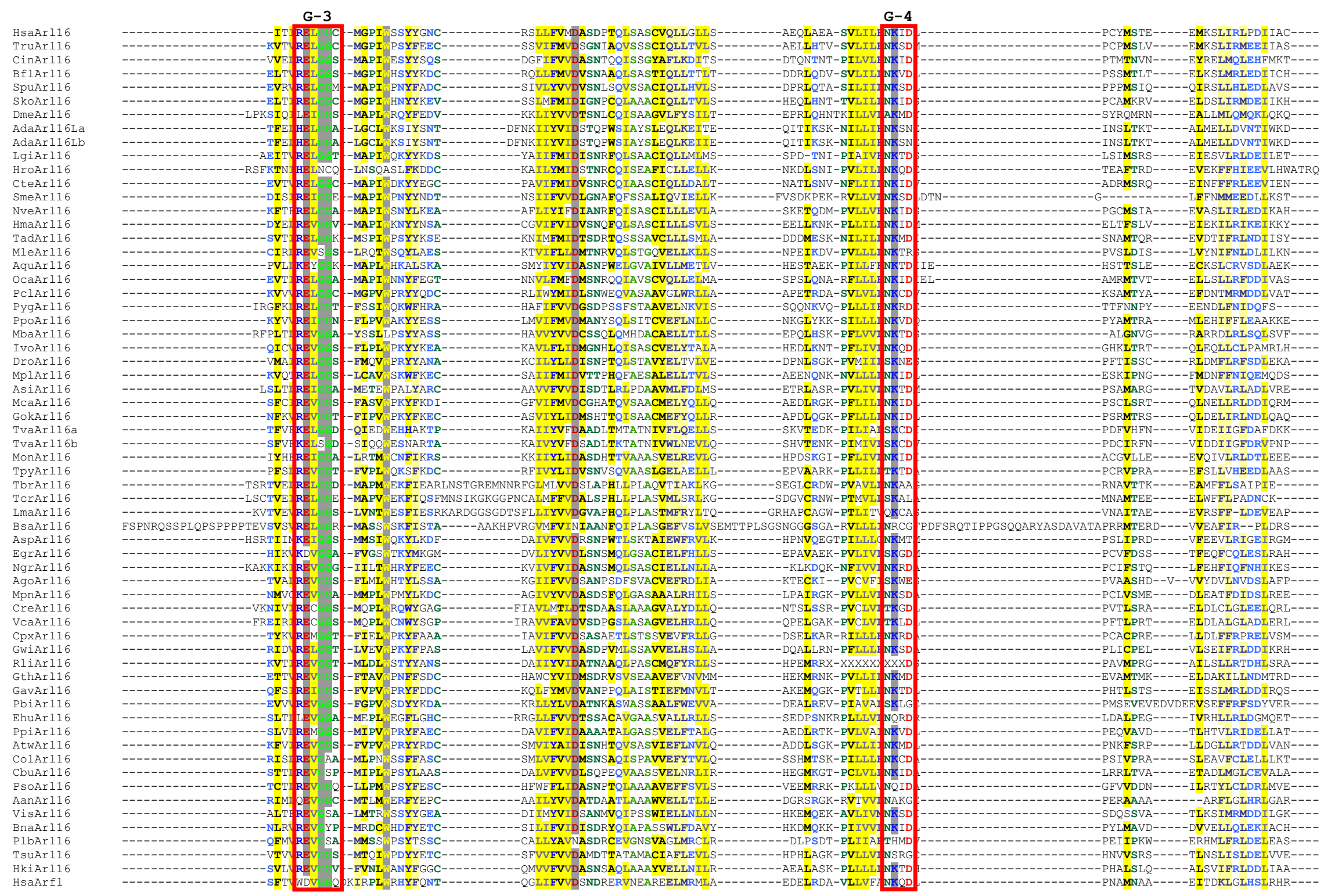




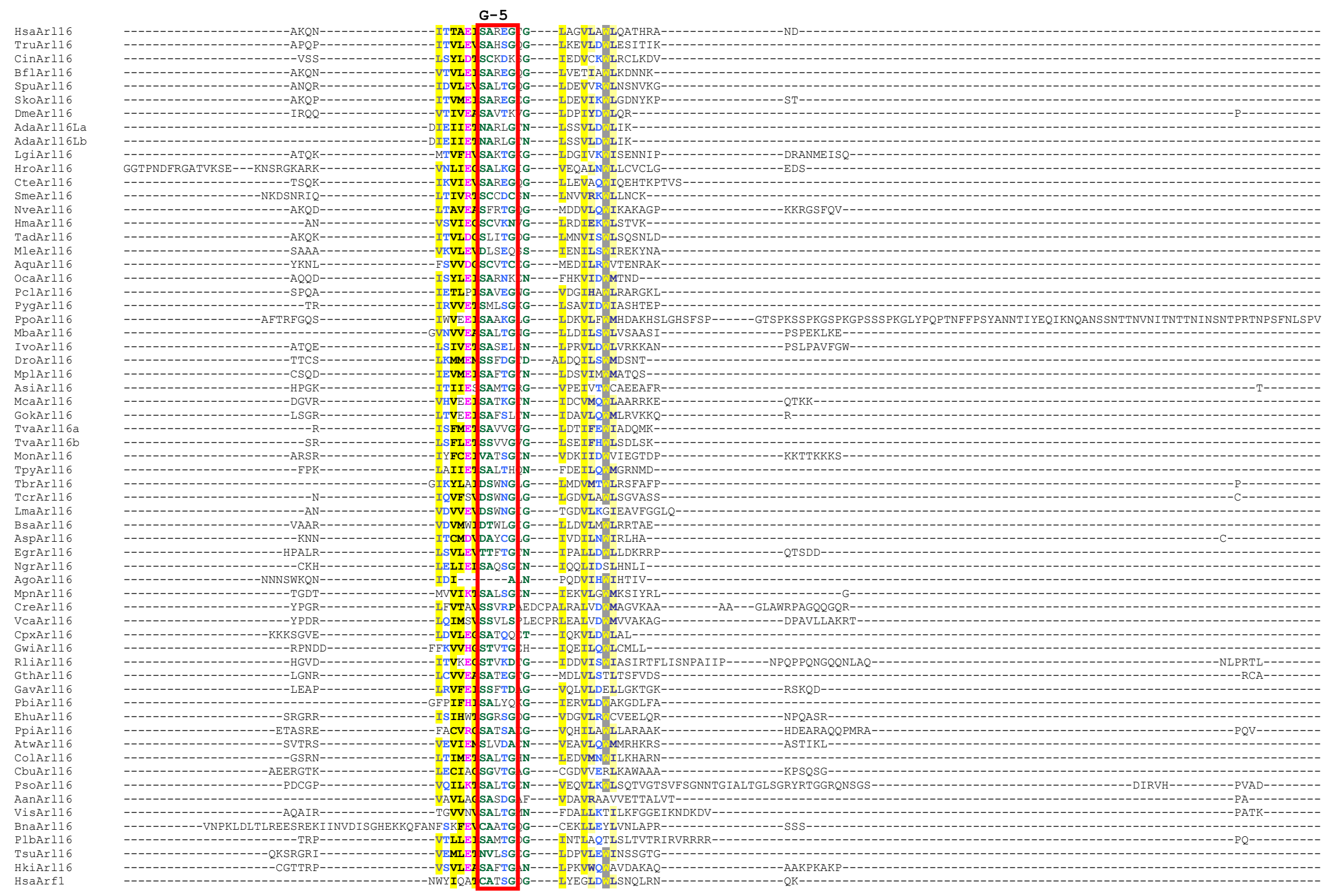


A

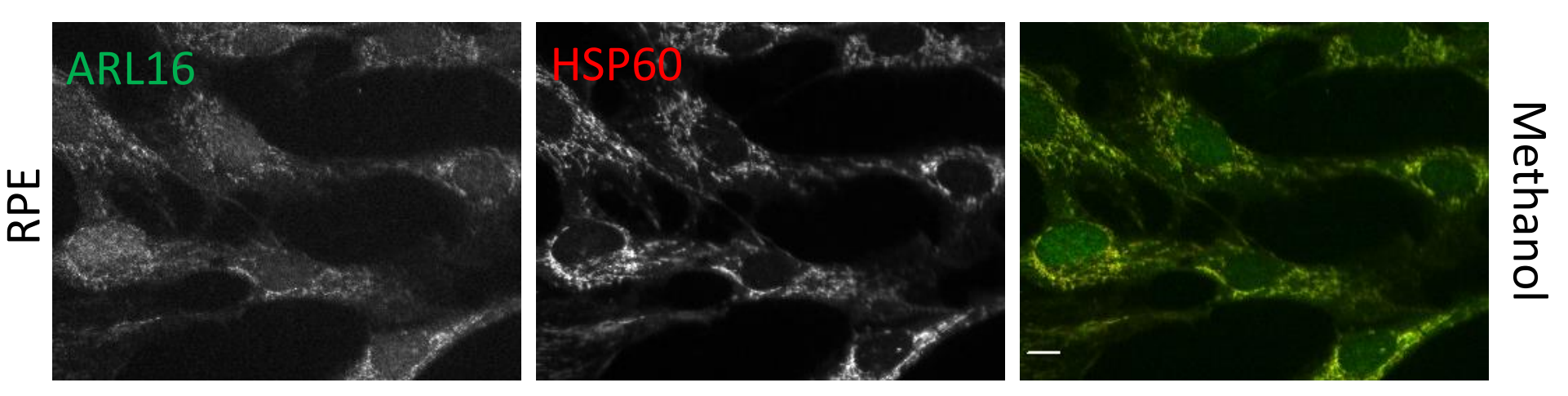

B

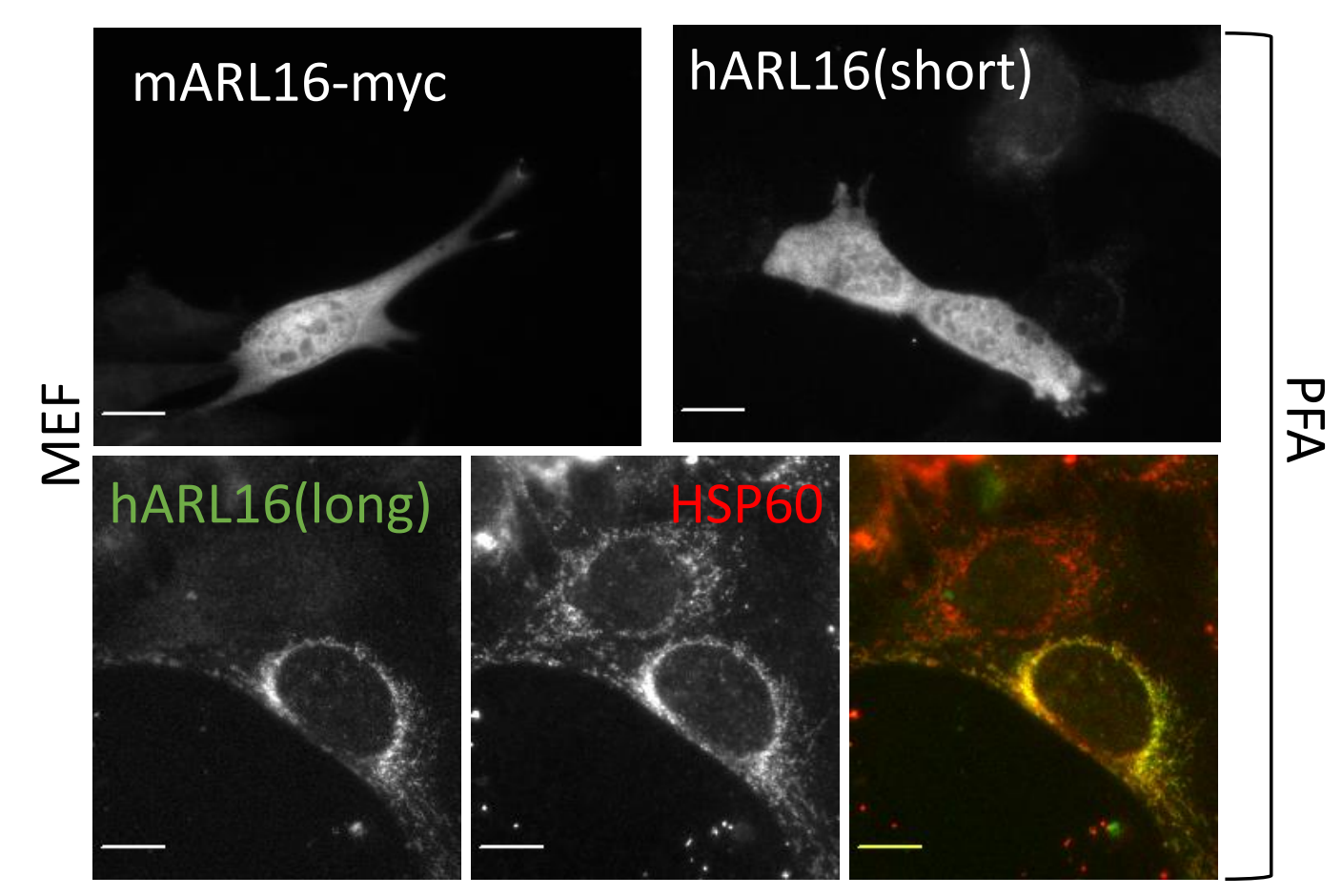

Figure S2 


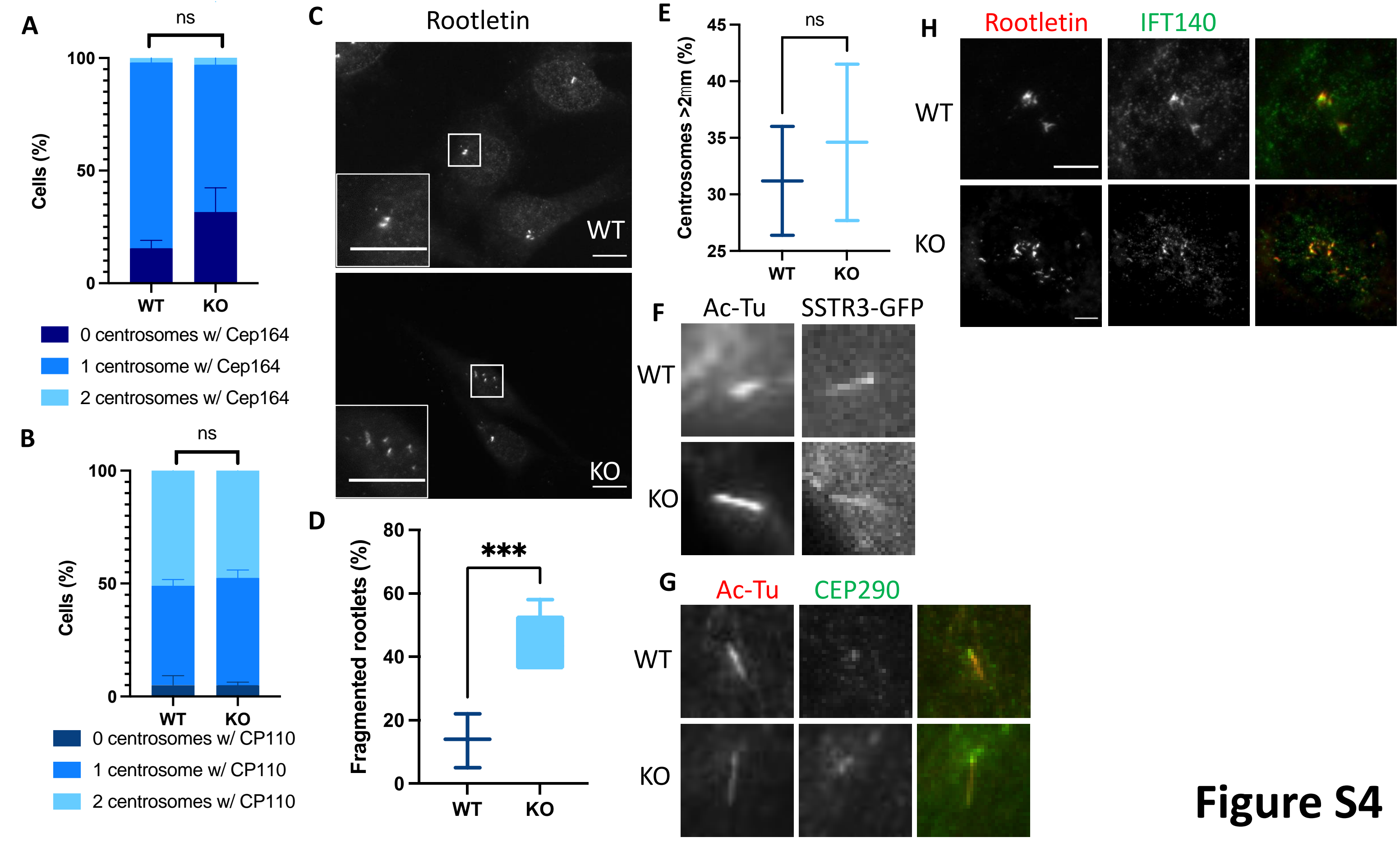




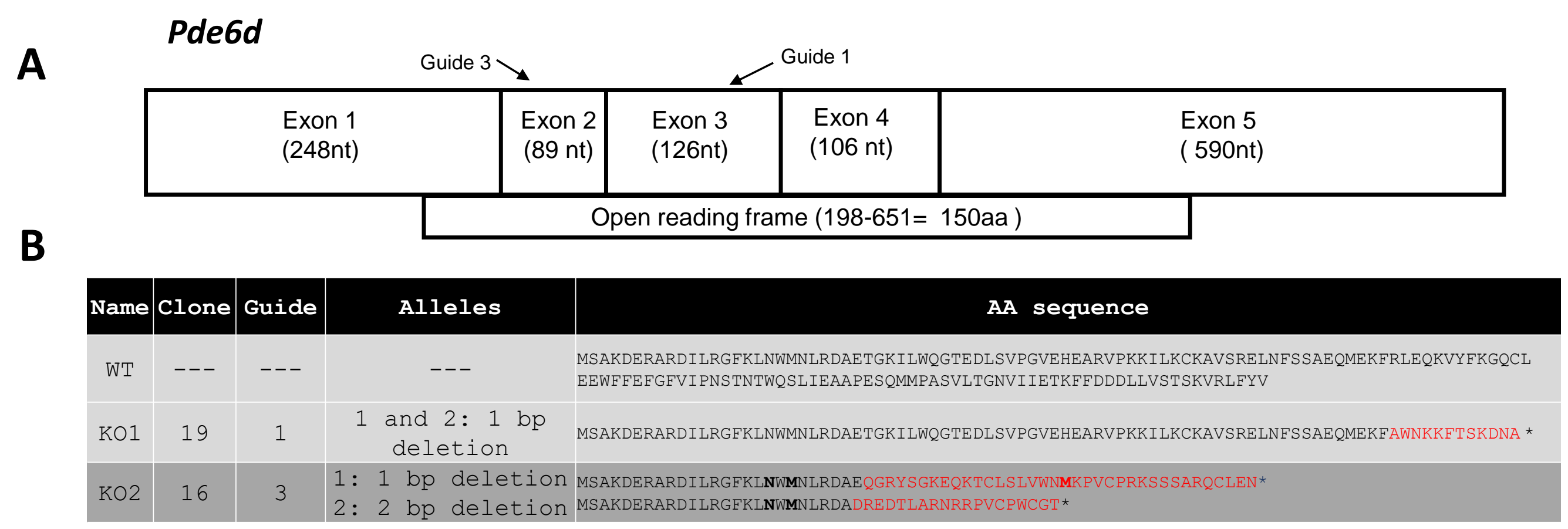

\section{Figure S5}

